\title{
Optimal trade-credit policy for perishable items deeming imperfect production and stock dependent demand
}

\author{
S. R. Singh and Swati Sharma*
}

Department of Mathematics, D. N. College, Meerut 250001, India

CHRONICLE ABSTRACT

\begin{tabular}{l}
\hline Article history: \\
Received June 12013 \\
Received in revised format \\
August 15 2013 \\
Accepted August 152013 \\
Available online \\
August 172013 \\
\hline Keywords: \\
Perishable items \\
Trade credit \\
Delay in payment
\end{tabular}

\begin{abstract}
Trade credit is the most succeeding economic phenomenon which is used by the supplier for encouraging the retailers to buy more quantity. In this article, a mathematical model with stock dependent demand and deterioration is developed to investigate the retailer's optimal inventory policy under the scheme of permissible delay in payment. It is assumed that defective items are produced during the production process and delay period is progressive. The objective is to minimize the total average cost of the system. To exemplify hypothesis of the proposed model numerical examples and sensitivity analysis are provided. Finally, the convexities of the cost functions and the effects of changing parameters are represented through the graphs.
\end{abstract}

(C) 2013 Growing Science Ltd. All rights reserved

\section{Introduction}

In long-established inventory models, it is often assumed that the purchasing cost for the items is paid by the retailer to the supplier as soon as the items have been received. In practice, a delay period known as trade credit period is offered by the supplier to the retailer, in paying for purchasing cost. Up to the end of the trade credit of a cycle, the retailer is free of charge, but he/she is charged on an interest for those items not being sold before this end. During the trade credit period, the retailer can accumulate revenues by selling items and earning interests. Goyal (1985) is the first person who developed the EOQ model under conditions of permissible delay in payments. Shah et al. (1988) studied the same model, incorporating shortages. Later on, Aggarwal and Jaggi (1995) discussed the inventory model considering deterioration and permissible delay in payment. Other motivating mechanisms in this research area are those of Teng (2002), Ouyang et al. (2006), Khanra et al. (2011), Singh et al. (2011), Teng et al. (2012), Singhal \& Singh (2013) and Singh and Sharma (2013).

\footnotetext{
* Corresponding author.

E-mail: jmlashi0@gmail.com (S. Sharma)

(c) 2014 Growing Science Ltd. All rights reserved. doi: $10.5267 / j$. .jiec.2013.08.002
} 
Deterioration of goods plays an important role in inventory system since in real life situations most of the physical goods deteriorate over time. Foods, pharmaceuticals, drugs, radioactive substances are some examples of items in which sufficient deterioration can take place during the normal cargo period and thus it plays an important role in analyzing the system. Generally, deterioration is defined as decay, damage or spoilage and obsolescence, which result in decrease of value of the original one. Ghare and Schrader (1963) presented the first model for decaying items. Covert and Philip (1973) extended their model considering Weibull distribution deterioration. Raafat (1991) presented a survey of literature on deteriorating inventory models. Hariga and Benkherouf (1994) proposed an inventory model for deteriorating items and later on Goyal and Giri (2001) provided a detailed review of deteriorating inventory literatures. Some other models dealing with the same issue are Yang and Wee (2006) and Kumar et al. (2012).

Many business practices reveal that the presence of a larger quantity of goods displayed attract customers to buy more quantity. This phenomenon implies that the demand may have a positive correlative with stock level. As Levin et al. (1972) observed that "large piles of consumer goods displayed in a supermarket will lead the customer to buy more. Yet, too much piled up in everyone's way leaves a negative impression on buyer and employee alike". Gupta and Vrat (1986) and Baker and Urban (1988) were the first to initiate a class of inventory models in which the demand rate is inventory dependent. Mandal and Phaujdar (1989) then developed a production inventory model for deteriorating items with uniform rate of production and linearly stock-dependent demand. Other papers related to this research area are by Zhou and Yang (2005), Lee and Dye (2012).

Most of the existing production inventory models ignored the presence of the imperfect production process. However, in real life situation, it is often observed that some of the items may be imperfect in nature, which are reworked at a cost to make them perfect. The production of defective items may be due to machine breakdown, labor problem, etc. Rosenblatt and Lee (1986) presumed that the time between the beginnings of the production run until the process goes out of control is exponential and that defective items can be reworked instantly at a cost and kept in stock. Kim and Hong (1999) determined the optimal production run length in deteriorating production processes.

Salameh and Jaber (2000) developed an economic production inventory model for items with imperfect quality items. Goyal and Barron (2002) extended the model presented by Salameh and Jaber's (2000). An inventory model is developed by Chung and Hou (2003) to obtain an optimal run time for a deteriorating production system with shortages. Yu et al. (2005) generalized the models of Salameh and Jaber (2000), considering deterioration and partial backordering. Later on, Kang (2010) presented an inventory model considering trade credit and items of imperfect quality. Recently, Sarkar and Moon (2011), Singh and Singh (2011), Sarkar (2012) and Singh et al. (2012) established some motivating inventory models with imperfect production processes.

An enormous work has been done in the field of trade-credit. Many previous economic order quantity inventory models are developed with trade-credit, a very few production inventory models are developed under allowable delay in payment. In addition, the inventory models for perishable items with imperfect production, stock dependent demand under trade-credit in which production rate depends on demand factor are much rare. Therefore, the present model is developed with these unique features. This model is an extension of the model Sarkar (2012) by considering deterioration and demand dependent production. The most favorable solution of the proposed model not only exists but also is unique. To obtain the optimal solution some lemmas are provided and with the help of sensitivity analysis, the effect of change in the parameters on the optimal policy is also disclosed.

\section{Assumptions and notations}


The following assumptions and notations are taken to discuss the model.

Assumptions

1. The inventory organism deals with a single type of items.

2. The replenishment rate is finite.

3. The delay in payment is offered to the retailer.

4. The demand is stock-dependent.

5. There is no repair or replacement of the deteriorated units.

6. Shortages are not permitted.

7. The lead time is zero.

8. The production of imperfect items is considered.

Notations

$I_{1}(t) \quad$ On-hand inventory at time $t$ where $0 \leq t \leq t_{1}$ (units)

$I_{2}(t) \quad$ On-hand inventory at time $\mathrm{t}$ where $t_{1} \leq t \leq T$ (units)

$p \quad$ Selling price per unit (\$/units)

$D \quad$ Stock-dependent demand i.e. $D=a+m I(t), a>0 ; m>0$ (units)

$T \quad$ Length of inventory cycle (year)

$P \quad$ Production rate (units per year), defined as $P=\mathrm{ka}$, and $\mathrm{k}>1$

$R \quad$ The $1^{\text {st }}$ offered trade-credit period without any charge (years)

$S \quad$ The $2^{\text {nd }}$ offered trade-credit period with charge (years)

$I_{e} \quad$ Rate of interest earned due to financing inventory (/year)

$I_{c 1} \quad$ Rate of interest charged due to the credit balance for $[R, S]$ (\$/year)

$I_{c 2} \quad$ Rate of interest charged due to the credit balance for $[S, T](\$ /$ year)

$C_{A} \quad$ Ordering cost per order (\$/order)

$C \quad$ Production cost (\$/unit)

$C_{d} \quad$ Deterioration cost (\$/unit)

$C_{h} \quad$ Holding cost (\$/unit item/unit time)

$C_{p} \quad$ Purchasing cost (\$/unit)

$C_{r} \quad$ Rework cost for the defective cost $(\$ /$ item)

$Z_{i} \quad$ Total cost of the system for $i=\{1,2,3\}(\$)$

\section{Formulation of the Model}

We consider an inventory model with stock-dependent demand model with different types of delay period. Depending on this policy, there may arise some cases:

Case (1): If the retailer pays the purchasing cost within the time $R$ (i.e., $T \leq R$ ), then there is no interest charged.

Case (2): If the retailer pays the purchasing cost after $R$ and before $\mathrm{S}$ (i.e., $R \leq T \leq S$ ), then the supplier can charge a rate of interest $I_{c 1}$ to the retailer. 
Case (3): If the retailer pays the purchasing cost after $\mathrm{S}$ and before $\mathrm{T}$ (i.e., $\mathrm{T} \geq \mathrm{S}$ ), the supplier can charge a rate of interest $I_{\mathrm{c} 2}$ on the unpaid balance (see Figs. 1-3).

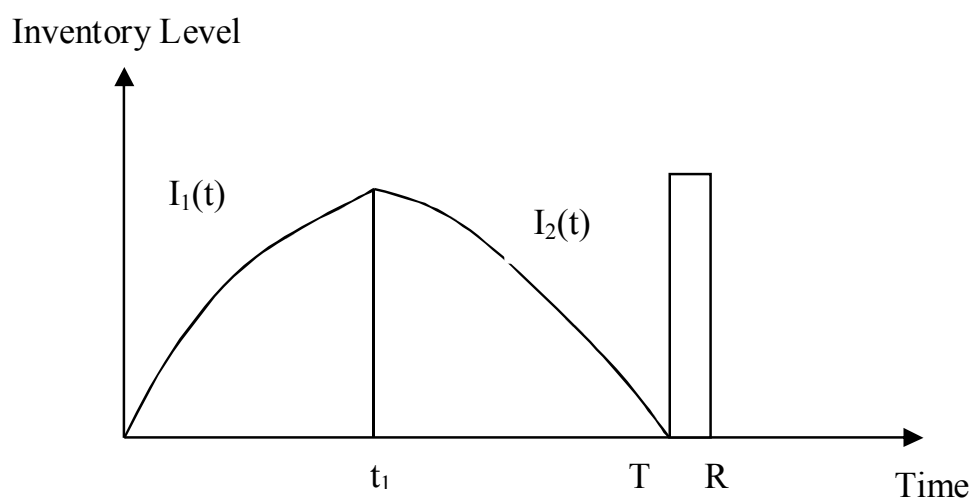

Fig. 1. Inventory versus time (Case-1: $T \leq R)$

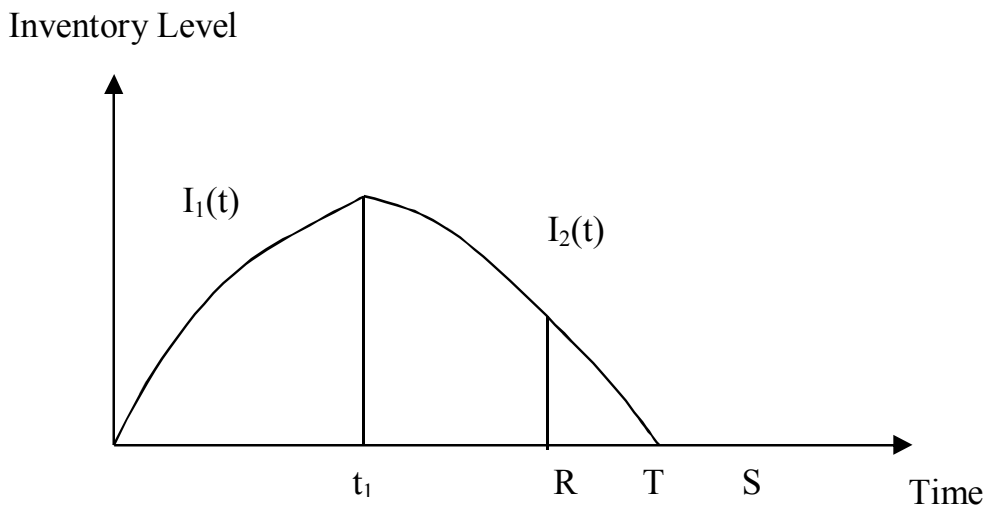

Fig. 2. Inventory versus time (Case-2: $R \leq T \leq S$ )

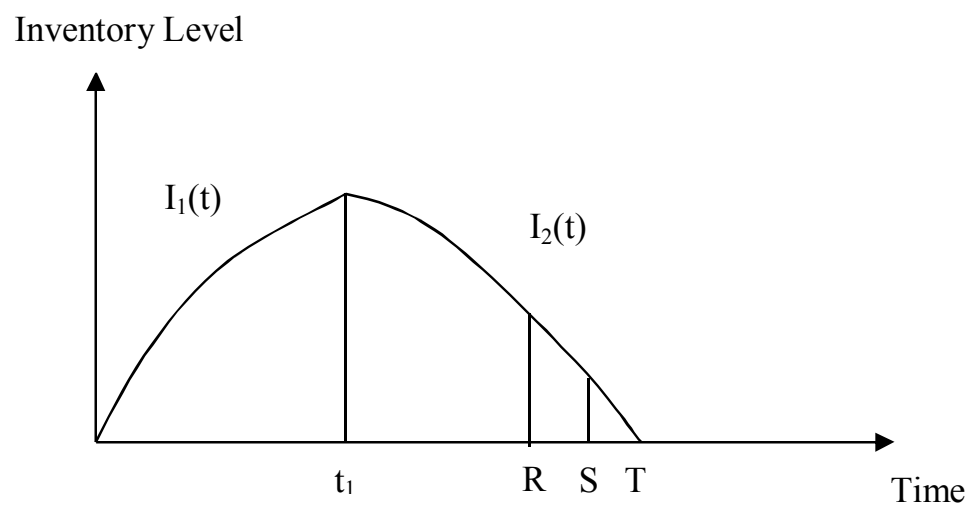

Fig. 3. Inventory versus time (Case-3: $T \geq S$ )

Now, the present state of the on-hand inventory is described by the following differential equations:

$I_{1}^{\prime}(t)=P-D\left(I_{1}(t)\right)-\theta I_{1}(t)=k a-\left(a+m I_{1}(t)\right)-\theta I_{1}(t), \quad 0 \leq \mathrm{t} \leq \mathrm{t}_{1}$

and 
$I_{2}{ }^{\prime}(t)=-D\left(I_{2}(t)\right)-\theta I_{2}(t)=-\left(a+m I_{2}(t)\right)-\theta I_{2}(t), \quad t_{1} \leq t \leq T$

with boundary conditions $I_{1}(0)=0$ and $I_{2}(T)=0$.

The solutions of the equations (1) and (2) are given as follows:

$$
I_{1}(t)=\frac{(k-1) a}{(m+\theta)}\left[1-e^{-(m+\theta) t}\right], \quad 0 \leq t \leq t_{1}
$$

and

$$
I_{2}(t)=\frac{a}{(m+\theta)}\left[e^{-(m+\theta)(t-T)}-1\right], \quad t_{1} \leq t \leq T
$$

Since $I_{1}\left(t_{1}\right)=I_{2}\left(t_{1}\right)$, we have

$$
\frac{(k-1) a}{(m+\theta)}\left[1-e^{-(m+\theta) t_{1}}\right]=\frac{a}{(m+\theta)}\left[e^{-(m+\theta)\left(t_{1}-T\right)}-1\right] \Rightarrow t_{1}=\frac{1}{(m+\theta)} \ln \left[1-\frac{\left(1-e^{(m+\theta) T}\right)}{k}\right] .
$$

Now, different costs of the inventory system are as follows:

Ordering cost is $\mathrm{OC}$ and is given by

$O C=\frac{C_{A}}{T}$.

Inventory holding cost is $\mathrm{HC}$ and is given by

$H C=\frac{C_{h}}{T}\left[\int_{0}^{t_{1}} I_{1}(t) d t+\int_{t_{1}}^{T} I_{2}(t) d t\right] \Rightarrow H C=\frac{C_{h}}{T}\left[\frac{a}{(m+\theta)^{2}}\left\{(k-1) e^{-(m+\theta) t_{1}}+e^{-(m+\theta)\left(t_{1}-T\right)}-k\right\}+\frac{a\left(k t_{1}-T\right)}{(m+\theta)}\right]$.

Deterioration cost for deteriorating items is $\mathrm{DC}$ and is given by

$D C=\frac{C_{d}}{T}\left[\int_{0}^{t_{1}} \theta I_{1}(t) d t+\int_{t_{1}}^{T} \theta I_{2}(t) d t\right] \Rightarrow D C=\frac{C_{d} \theta}{T}\left[\frac{a}{(m+\theta)^{2}}\left\{(k-1) e^{-(m+\theta) t_{1}}+e^{-(m+\theta)\left(t_{1}-T\right)}-k\right\}+\frac{a\left(k t_{1}-T\right)}{(m+\theta)}\right]$.

Production cost is PRC and is given by

$P R C=\frac{C}{T} \int_{0}^{t_{1}} P d t=\frac{C k a t_{1}}{T}$.

Purchasing cost is PUC and is given by

$P U C=\frac{C_{p}}{T} \int_{0}^{t_{1}} P d t=\frac{C_{p} k a t_{1}}{T}$.

Along with the trade credit, the paper considers the production of imperfect items. The lifetime of defective item follows a Weibull distribution defined as $\psi(t)=\alpha t^{\beta}, \beta>-1$, where $\alpha$, $\beta$ are two parameters and $\mathrm{t}$ is the time to failure. Hence, the total number of defective items is:

$N=\int_{0}^{t_{1}} P \psi(t) e^{\left[-\int_{0}^{t} \psi(\tau) d \tau\right]} d t=k a\left(1-e^{-\left(\frac{\alpha}{\beta+1}\right) t_{1}^{\beta+1}}\right)$.

The rework cost is $\mathrm{RC}$ and is given by

$R C=\frac{C_{r} N}{T}=\frac{C_{r} k a}{T}\left(1-e^{-\left(\frac{\alpha}{\beta+1}\right)_{t_{1}}^{\beta+1}}\right)$.

Now, for different delay periods:

Case (1): $T \leq R$

In this case, interest earned is $\mathrm{IE}_{1}$ and is given by

$I E_{1}=\frac{p I_{e}}{T}\left[\int_{0}^{t_{1}}\left(t_{1}-t\right) D\left(I_{1}(t)\right) d t+\int_{t_{1}}^{T}(T-t) D\left(I_{2}(t)\right) d t+(R-T)\left\{\int_{0}^{t_{1}} D\left(I_{1}(t)\right) d t+\int_{t_{1}}^{T} D\left(I_{2}(t)\right) d t\right\}\right]$. 


$$
\begin{aligned}
& I E_{1}=\frac{p I_{e}}{T}\left[\left(R-T+t_{1}\right)\left\{a t_{1}+\frac{m(k-1) a t_{1}}{(m+\theta)}+\frac{m(k-1) a}{(m+\theta)^{2}}\left(e^{-(m+\theta) t_{1}}-1\right)\right\}+R\left\{a\left(T-t_{1}\right)+\frac{m a}{(m+\theta)^{2}}\left(e^{-(m+\theta)\left(t_{1}-T\right)}-1\right)\right.\right. \\
&\left.-\frac{m a\left(T-t_{1}\right)}{(m+\theta)}\right\}-\left\{\frac{a t_{1}^{2}}{2}+\frac{m(k-1) a t_{1}^{2}}{2(m+\theta)}+\frac{m(k-1) a t_{1} e^{-(m+\theta) t_{1}}}{(m+\theta)^{2}}+\frac{m(k-1) a}{(m+\theta)^{3}}\left(e^{-(m+\theta) t_{1}}-1\right)\right\} \\
&-\left\{\frac{a\left(T^{2}-t_{1}^{2}\right)}{2}+\frac{m a}{(m+\theta)^{2}}\left(t_{1} e^{-(m+\theta)\left(t_{1}-T\right)}-T\right)+\frac{m a}{(m+\theta)^{3}}\left(e^{-(m+\theta)\left(t_{1}-T\right)}-1\right)-\frac{m a\left(T^{2}-t_{1}^{2}\right)}{2(m+\theta)}\right\} .
\end{aligned}
$$

In this case, interest charged is $\mathrm{IC}_{1}$ and is given by

$$
I C_{1}=0 \text {. }
$$

Case (2): $\mathrm{R} \leq \mathrm{T} \leq \mathrm{S}$

In this case, interest earned is $\mathrm{IE}_{2}$ and is given by

$$
\begin{aligned}
I E_{2}=\frac{p I_{e}}{T} & {\left[\int_{0}^{t_{1}}\left(t_{1}-t\right) D\left(I_{1}(t)\right) d t+\int_{t_{1}}^{T}(T-t) D\left(I_{2}(t)\right) d t\right] . } \\
I E_{2}=\frac{p I_{e}}{T}[ & {\left[t_{1}\left\{a t_{1}+\frac{m(k-1) a t_{1}}{(m+\theta)}+\frac{m(k-1) a}{(m+\theta)^{2}}\left(e^{-(m+\theta) t_{1}}-1\right)\right\}+T\left\{a\left(T-t_{1}\right)+\frac{m a}{(m+\theta)^{2}}\left(e^{-(m+\theta)\left(t_{1}-T\right)}-1\right)-\frac{m a\left(T-t_{1}\right)}{(m+\theta)}\right\}\right.} \\
& -\left\{\frac{a t_{1}^{2}}{2}+\frac{m(k-1) a t_{1}^{2}}{2(m+\theta)}+\frac{m(k-1) a t_{1} e^{-(m+\theta) t_{1}}}{(m+\theta)^{2}}+\frac{m(k-1) a}{(m+\theta)^{3}}\left(e^{-(m+\theta) t_{1}}-1\right)\right\} \\
& -\left\{\frac{a\left(T^{2}-t_{1}^{2}\right)}{2}+\frac{m a}{(m+\theta)^{2}}\left(t_{1} e^{-(m+\theta)\left(t_{1}-T\right)}-T\right)+\frac{m a}{(m+\theta)^{3}}\left(e^{-(m+\theta)\left(t_{1}-T\right)}-1\right)-\frac{m a\left(T^{2}-t_{1}^{2}\right)}{2(m+\theta)}\right\} .
\end{aligned}
$$

In this case, interest charged is $\mathrm{IC}_{2}$ and is given by

$$
I C_{2}=\frac{I_{c 1} C_{P}}{T} \int_{R}^{T} I_{2}(t) d t=\frac{I_{c 1} C_{P} a}{T(m+\theta)}\left[R-T-\frac{1}{(m+\theta)}+\frac{e^{-(m+\theta)(R-T)}}{(m+\theta)}\right] .
$$

Case (3): $T \geq S$

In this case, interest earned is $\mathrm{IE}_{3}$ and is given by

$$
\begin{aligned}
& I E_{3}= \frac{p I_{e}}{T}\left[\int_{0}^{t_{1}}\left(t_{1}-t\right) D\left(I_{1}(t)\right) d t+\int_{t_{1}}^{S}(T-t) D\left(I_{2}(t)\right) d t\right] . \\
& I E_{3}=\frac{p I_{e}}{T}\left[t_{1}\left\{a t_{1}+\frac{m(k-1) a t_{1}}{(m+\theta)}+\frac{m(k-1) a}{(m+\theta)^{2}}\left(e^{-(m+\theta) t_{1}}-1\right)\right\}+T\left\{a\left(S-t_{1}\right)+\frac{m a}{(m+\theta)^{2}}\left(e^{-(m+\theta)\left(t_{1}-T\right)}-e^{-(m+\theta)(S-T)}\right)\right.\right. \\
&\left.-\frac{m a\left(S-t_{1}\right)}{(m+\theta)}\right\}-\left\{\frac{a t_{1}^{2}}{2}+\frac{m(k-1) a t_{1}^{2}}{2(m+\theta)}+\frac{m(k-1) a t_{1} e^{-(m+\theta) t_{1}}}{(m+\theta)^{2}}+\frac{m(k-1) a}{(m+\theta)^{3}}\left(e^{-(m+\theta) t_{1}}-1\right)\right\} \\
&-\left\{\frac{a\left(S^{2}-t_{1}^{2}\right)}{2}+\frac{m a}{(m+\theta)^{2}}\left(t_{1} e^{-(m+\theta)\left(t_{1}-T\right)}-S e^{-(m+\theta)(S-T)}\right)+\frac{m a}{(m+\theta)^{3}}\left(e^{-(m+\theta)\left(t_{1}-T\right)}-e^{-(m+\theta)(S-T)}\right)-\frac{m a\left(S^{2}-t_{1}^{2}\right)}{2(m+\theta)}\right\} .
\end{aligned}
$$

In this case, interest charged is $\mathrm{IC}_{3}$ and is given by

$$
I C_{3}=\frac{I_{c 2} C_{P}}{T} \int_{S}^{T} I_{2}(t) d t=\frac{I_{c 2} C_{P} a}{T(m+\theta)}\left[S-T-\frac{1}{(m+\theta)}+\frac{e^{-(m+\theta)(S-T)}}{(m+\theta)}\right] .
$$

Thus, the total average cost for case (1): is $\mathrm{Z}_{1}(\mathrm{~T})$ and is given by,

$$
Z_{1}(T)=O C+H C+D C+P R C+P U C+R C+I C_{1}-I E_{1} .
$$

The total average cost for case (2) is $Z_{2}(T)$ and is given by 
$Z_{2}(T)=O C+H C+D C+P R C+P U C+R C+I C_{2}-I E_{2}$.

The total average cost for case $(3)$ is $Z_{3}(T)$ and is given by

$Z_{3}(T)=O C+H C+D C+P R C+P U C+R C+I C_{3}-I E_{3}$.

Our objective is to minimize the total cost of the inventory system. The necessary conditions for the existence of the optimal solutions are

$$
\begin{aligned}
& \frac{d Z_{1}(T)}{d T}=0, \\
& \frac{d Z_{2}(T)}{d T}=0, \\
& \frac{d Z_{3}(T)}{d T}=0 .
\end{aligned}
$$

Using the software Mathematica-8.0, from eq. (22) to Eq. (24) we can determine the optimum values of $T=T_{i}{ }^{*}$, where $i=1,2,3$ and the optimal value $Z_{i}\left(T_{i}{ }^{*}\right)$, where $i=1,2,3$ of the total cost can be determined by (21) provided they satisfy the sufficiency conditions for minimizing $Z_{i}\left(T_{i}{ }^{*}\right)$, where $i=1,2,3$ given by $\left.\frac{d^{2} Z_{i}(T)}{d T^{2}}\right|_{T=T_{i}^{*}}>0$, where $i=1,2,3$

For the cost minimization we may formulate the three lemmas (motivated by Sarkar (2012)) as follows:

Lemma 1. $Z_{l}\left(T^{*}\right)$ must have a minimum value at $T^{*}$ if it satisfies the equation $T^{*}=\frac{\left[C_{A}+\phi_{2}\left(C_{p}+C\right)+\phi_{4} C_{r} k a+\phi_{1}\left(C_{h}+\theta C_{d}\right)-\phi_{3} p I_{e}\right]}{\left[\phi_{5}\left(C_{h}+\theta C_{d}\right)+\phi_{11}\left(C_{p}+C\right)+\phi_{10} C_{r} k a-\phi_{6} p I_{e}\right]}$ and the inequality $\phi_{16}\left(T^{*}\right)^{2}+\phi_{17} T^{*}+\phi_{18}>0$ where all the values of $\phi_{\mathrm{i}}$ 's are given in Appendix 1.

Proof. For minimization of the total cost $Z_{1},\left.\frac{d Z_{1}}{d T}\right|_{T=T^{*}}=0$ and $\left.\frac{d^{2} Z_{1}}{d T^{2}}\right|_{T=T^{*}}>0$ must be satisfied.

Now,

$$
\begin{aligned}
Z_{1}= & \frac{C_{A}}{T}+\frac{\left(C_{h}+\theta C_{d}\right)}{T}\left\{\frac{a}{(m+\theta)^{2}}\left\{(k-1) e^{-(m+\theta) t_{1}}+e^{-(m+\theta)\left(t_{1}-T\right)}-k\right\}+\frac{a\left(k t_{1}-T\right)}{(m+\theta)}\right\}+\frac{\left(C_{p}+C\right) k a t_{1}}{T} \\
& -\frac{p I_{e}}{T}\left[\left(R-T+t_{1}\right)\left\{a t_{1}+\frac{m(k-1) a t_{1}}{(m+\theta)}+\frac{m(k-1) a}{(m+\theta)^{2}}\left(e^{-(m+\theta) t_{4}}-1\right)\right\}+R\left\{a\left(T-t_{1}\right)+\frac{m a}{(m+\theta)^{2}}\left(e^{-(m+\theta)\left(t_{1}-T\right)}-1\right)\right.\right. \\
& \left.-\frac{m a\left(T-t_{1}\right)}{(m+\theta)}\right\}-\left\{\frac{a t_{1}^{2}}{2}+\frac{m(k-1) a t_{1}^{2}}{2(m+\theta)}+\frac{m(k-1) a t_{1} e^{-(m+\theta) t_{1}}}{(m+\theta)^{2}}+\frac{m(k-1) a}{(m+\theta)^{3}}\left(e^{-(m+\theta) t_{1}}-1\right)\right\} \\
& \left.-\left\{\frac{a\left(T^{2}-t_{1}^{2}\right)}{2}+\frac{m a}{(m+\theta)^{2}}\left(t_{1} e^{-(m+\theta)\left(t_{1}-T\right)}-T\right)+\frac{m a}{(m+\theta)^{3}}\left(e^{-(m+\theta)\left(t_{1}-T\right)}-1\right)-\frac{m a\left(T^{2}-t_{1}^{2}\right)}{2(m+\theta)}\right\}\right]+\frac{C_{r} k a}{T}\left(1-e^{-\left(\frac{\alpha}{\beta+1}\right)^{\beta_{1}+t_{1}}}\right) .
\end{aligned}
$$

Differentiating the above expression with respect to $\mathrm{T}$, we get

$$
\frac{d Z_{1}}{d T}=\frac{\phi_{5}\left(C_{h}+\theta C_{d}\right)}{T}-\frac{\phi_{1}\left(C_{h}+\theta C_{d}\right)}{T^{2}}-\frac{C_{A}}{T^{2}}-\frac{\phi_{2}\left(C_{p}+C\right)}{T^{2}}+\frac{\phi_{11}\left(C_{p}+C\right)}{T}+\frac{\phi_{3} p I_{e}}{T^{2}}-\frac{\phi_{6} p I_{e}}{T}-\frac{\phi_{4} C_{r} k a}{T^{2}}+\frac{\phi_{10} C_{r} k a}{T},
$$

and again, differentiating the above expression with respect to $\mathrm{T}$, we find

$$
\begin{aligned}
\frac{d^{2} Z_{1}}{d T^{2}}= & \frac{2 C_{A}}{T^{3}}+\frac{2 \phi_{1}\left(C_{h}+\theta C_{d}\right)}{T^{3}}-\frac{2 \phi_{5}\left(C_{h}+\theta C_{d}\right)}{T^{2}}+\frac{\phi_{12}\left(C_{h}+\theta C_{d}\right)}{T}+\frac{2 \phi_{2}\left(C_{p}+C\right)}{T^{3}}-\frac{2 \phi_{11}\left(C_{p}+C\right)}{T^{2}} \\
& +\frac{\phi_{13}\left(C_{p}+C\right)}{T}-\frac{2 \phi_{3} p I_{e}}{T^{3}}+\frac{2 \phi_{6} p I_{e}}{T^{2}}-\frac{\phi_{14} p I_{e}}{T}+\frac{2 \phi_{4} C_{r} k a}{T^{3}}-\frac{2 \phi_{10} C_{r} k a}{T^{2}}+\frac{\phi_{15} C_{r} k a}{T},
\end{aligned}
$$


For minimum of $Z_{1},\left.\frac{d Z_{1}}{d T}\right|_{T=T^{*}}=0$ and $\left.\frac{d^{2} Z_{1}}{d T^{2}}\right|_{T=T^{*}}>0$ imply that

$\left[\frac{\phi_{5}\left(C_{h}+\theta C_{d}\right)}{T}+\frac{\phi_{11}\left(C_{p}+C\right)}{T}+\frac{\phi_{3} p I_{e}}{T^{2}}+\frac{\phi_{10} C_{r} k a}{T}\right]_{T=T^{*}}=\left[\frac{C_{A}}{T^{2}}+\frac{\phi_{1}\left(C_{h}+\theta C_{d}\right)}{T^{2}}+\frac{\phi_{2}\left(C_{p}+C\right)}{T^{2}}+\frac{\phi_{6} p I_{e}}{T}+\frac{\phi_{4} C_{r} k a}{T^{2}}\right]_{T=T^{*}}$,

and

$\left[\frac{2 C_{A}}{T^{3}}+\frac{2 \phi_{1}\left(C_{h}+\theta C_{d}\right)}{T^{3}}+\frac{\phi_{12}\left(C_{h}+\theta C_{d}\right)}{T}+\frac{2 \phi_{2}\left(C_{p}+C\right)}{T^{3}}+\frac{\phi_{13}\left(C_{p}+C\right)}{T}+\frac{2 \phi_{6} p I_{e}}{T^{2}}+\frac{2 \phi_{4} C_{r} k a}{T^{3}}+\frac{\phi_{15} C_{r} k a}{T}\right]_{T=T^{*}}$

$>\left[\frac{2 \phi_{5}\left(C_{h}+\theta C_{d}\right)}{T^{2}}+\frac{2 \phi_{11}\left(C_{p}+C\right)}{T^{2}}+\frac{2 \phi_{3} p I_{e}}{T^{3}}+\frac{\phi_{14} p I_{e}}{T}+\frac{2 \phi_{10} C_{r} k a}{T^{2}}\right]_{T=T^{*}}$.

After some simplification, we get

$T^{*}=\frac{\left[C_{A}+\phi_{2}\left(C_{p}+C\right)+\phi_{4} C_{r} k a+\phi_{1}\left(C_{h}+\theta C_{d}\right)-\phi_{3} p I_{e}\right]}{\left[\phi_{5}\left(C_{h}+\theta C_{d}\right)+\phi_{11}\left(C_{p}+C\right)+\phi_{10} C_{r} k a-\phi_{6} p I_{e}\right]}$ and $\phi_{16}\left(T^{*}\right)^{2}+\phi_{17} T^{*}+\phi_{18}>0$.

Hence the proof.

Lemma 2. $\mathrm{Z}_{2}\left(\mathrm{~T}^{*}\right)$ must have a minimum value at $\mathrm{T}^{*}$ if it satisfies the equation $T^{*}=\frac{\left[C_{A}+\phi_{1}\left(C_{h}+\theta C_{d}\right)+\phi_{2}\left(C_{p}+C\right)+\phi_{4} C_{r} k a+\phi_{21} C_{p} I_{c 1}-\phi_{19} p I_{e}\right]}{\left[\phi_{5}\left(C_{h}+\theta C_{d}\right)+\phi_{11}\left(C_{p}+C\right)+\phi_{10} C_{r} k a+\phi_{22} C_{p} I_{c 1}-\phi_{20} p I_{e}\right]}$ and the inequality $\phi_{25}\left(T^{*}\right)^{2}+\phi_{26} T^{*}+\phi_{27}>0$ where all the values of $\phi_{\mathrm{i}}$ 's are given in Appendix 2.

Proof. For minimization of the total cost $Z_{2},\left.\frac{d Z_{2}}{d T}\right|_{T=T^{*}}=0$ and $\left.\frac{d^{2} Z_{2}}{d T^{2}}\right|_{T=T^{*}}>0$ must be satisfied.

Now,

$$
\begin{aligned}
Z_{2}= & \frac{C_{A}}{T}+\frac{\left(C_{h}+\theta C_{d}\right)}{T}\left\{\frac{a}{(m+\theta)^{2}}\left\{(k-1) e^{-(m+\theta) t_{1}}+e^{-(m+\theta)\left(t_{1}-T\right)}-k\right\}+\frac{a\left(k t_{1}-T\right)}{(m+\theta)}\right\}+\frac{\left(C_{p}+C\right) k a t_{1}}{T} \\
& -\frac{p I_{e}}{T}\left[t_{1}\left\{a t_{1}+\frac{m(k-1) a t_{1}}{(m+\theta)}+\frac{m(k-1) a}{(m+\theta)^{2}}\left(e^{-(m+\theta))_{1}}-1\right)\right\}+T\left\{a\left(T-t_{1}\right)+\frac{m a}{(m+\theta)^{2}}\left(e^{-(m+\theta)\left(t_{1}-T\right)}-1\right)\right.\right. \\
& \left.-\frac{m a\left(T-t_{1}\right)}{(m+\theta)}\right\}-\left\{\frac{a t_{1}^{2}}{2}+\frac{m(k-1) a t_{1}^{2}}{2(m+\theta)}+\frac{m(k-1) a t_{1} e^{-(m+\theta) t_{1}}}{(m+\theta)^{2}}+\frac{m(k-1) a}{(m+\theta)^{3}}\left(e^{-(m+\theta) t_{1}}-1\right)\right\} \\
& \left.-\left\{\frac{a\left(T^{2}-t_{1}^{2}\right)}{2}+\frac{m a}{(m+\theta)^{2}}\left(t_{1} e^{-(m+\theta)\left(t_{1}-T\right)}-T\right)+\frac{m a}{(m+\theta)^{3}}\left(e^{-(m+\theta)\left(t_{1}-T\right)}-1\right)-\frac{m a\left(T^{2}-t_{1}^{2}\right)}{2(m+\theta)}\right\}\right] \\
& +\frac{C_{r} k a}{T}\left(1-e^{-\left(\frac{\alpha}{\beta+1}\right) t_{1} t^{\beta+1}}\right)+\frac{I_{c 1} C_{P} a}{T(m+\theta)}\left\{R-T-\frac{1}{(m+\theta)}+\frac{e^{-(m+\theta)(R-T)}}{(m+\theta)}\right\} .
\end{aligned}
$$

Differentiating the above expression with respect to $\mathrm{T}$, we get $\frac{d Z_{2}}{d T}=\frac{\phi_{5}\left(C_{h}+\theta C_{d}\right)}{T}-\frac{\phi_{1}\left(C_{h}+\theta C_{d}\right)}{T^{2}}-\frac{C_{A}}{T^{2}}-\frac{\phi_{2}\left(C_{p}+C\right)}{T^{2}}+\frac{\phi_{11}\left(C_{p}+C\right)}{T}+\frac{\phi_{19} p I_{e}}{T^{2}}-\frac{\phi_{20} p I_{e}}{T}-\frac{\phi_{4} C_{r} k a}{T^{2}}+\frac{\phi_{10} C_{r} k a}{T}-\frac{\phi_{21} C_{p} I_{c 1}}{T^{2}}+\frac{\phi_{22} C_{p} I_{c 1}}{T}$, and again, differentiating the above expression with respect to $T$, we have

$$
\begin{aligned}
\frac{d^{2} Z_{2}}{d T^{2}}= & \frac{2 C_{A}}{T^{3}}+\frac{2 \phi_{1}\left(C_{h}+\theta C_{d}\right)}{T^{3}}-\frac{2 \phi_{5}\left(C_{h}+\theta C_{d}\right)}{T^{2}}+\frac{\phi_{12}\left(C_{h}+\theta C_{d}\right)}{T}+\frac{2 \phi_{2}\left(C_{p}+C\right)}{T^{3}}-\frac{2 \phi_{11}\left(C_{p}+C\right)}{T^{2}}+\frac{\phi_{13}\left(C_{p}+C\right)}{T} \\
& -\frac{2 \phi_{19} p I_{e}}{T^{3}}+\frac{2 \phi_{20} p I_{e}}{T^{2}}-\frac{\phi_{23} p I_{e}}{T}+\frac{2 \phi_{4} C_{r} k a}{T^{3}}-\frac{2 \phi_{10} C_{r} k a}{T^{2}}+\frac{\phi_{15} C_{r} k a}{T}+\frac{2 \phi_{21} C_{p} I_{c 1}}{T^{3}}-\frac{2 \phi_{22} C_{p} I_{c 1}}{T^{2}}+\frac{\phi_{24} C_{p} I_{c 1}}{T} .
\end{aligned}
$$


For minimum of $Z_{2},\left.\frac{d Z_{2}}{d T}\right|_{T=T^{*}}=0$ and $\left.\frac{d^{2} Z_{2}}{d T^{2}}\right|_{T=T^{*}}>0$ imply that

$\left[\frac{\phi_{5}\left(C_{h}+\theta C_{d}\right)}{T}+\frac{\phi_{11}\left(C_{p}+C\right)}{T}+\frac{\phi_{9} p I_{e}}{T^{2}}+\frac{\phi_{10} C_{r} k a}{T}+\frac{\phi_{22} C_{p} I_{c 1}}{T}\right]_{T=T^{*}}=\left[\frac{C_{A}}{T^{2}}+\frac{\phi_{1}\left(C_{h}+\theta C_{d}\right)}{T^{2}}+\frac{\phi_{2}\left(C_{p}+C\right)}{T^{2}}+\frac{\phi_{20} p I_{e}}{T}+\frac{\phi_{4} C_{r} k a}{T^{2}}+\frac{\phi_{21} C_{p} I_{c 1}}{T^{2}}\right]_{T=T^{*}}$,

and

$\left[\frac{2 C_{A}}{T^{3}}+\frac{2 \phi_{1}\left(C_{h}+\theta C_{d}\right)}{T^{3}}+\frac{\phi_{12}\left(C_{h}+\theta C_{d}\right)}{T}+\frac{2 \phi_{2}\left(C_{p}+C\right)}{T^{3}}+\frac{\phi_{3}\left(C_{p}+C\right)}{T}+\frac{2 \phi_{20} p I_{e}}{T^{2}}+\frac{2 \phi_{4} C_{r} k a}{T^{3}}+\frac{\phi_{15} C_{r} k a}{T}+\frac{2 \phi_{21} C_{p} I_{c 1}}{T^{3}}+\frac{\phi_{24} C_{p} I_{c 1}}{T}\right]_{T=I^{\prime}}$

$>\left[\frac{2 \phi_{5}\left(C_{h}+\theta C_{d}\right)}{T^{2}}+\frac{2 \phi_{11}\left(C_{p}+C\right)}{T^{2}}+\frac{2 \phi_{19} p I_{e}}{T^{3}}+\frac{\phi_{23} p I_{e}}{T}+\frac{2 \phi_{10} C_{r} k a}{T^{2}}+\frac{2 \phi_{22} C_{p} I_{c 1}}{T^{2}}\right]_{T=T^{*}}$.

After some simplification, we get

$T^{*}=\frac{\left[C_{A}+\phi_{1}\left(C_{h}+\theta C_{d}\right)+\phi_{2}\left(C_{p}+C\right)+\phi_{4} C_{r} k a+\phi_{21} C_{p} I_{c 1}-\phi_{19} p I_{e}\right]}{\left[\phi_{5}\left(C_{h}+\theta C_{d}\right)+\phi_{11}\left(C_{p}+C\right)+\phi_{10} C_{r} k a+\phi_{22} C_{p} I_{c 1}-\phi_{20} p I_{e}\right]}$ and $\phi_{25}\left(T^{*}\right)^{2}+\phi_{26} T^{*}+\phi_{27}>0$

Hence the proof.

Lemma 3. $\mathrm{Z}_{3}\left(\mathrm{~T}^{*}\right)$ must have a minimum value at $\mathrm{T}^{*}$ if it satisfies the equation $T^{*}=\frac{\left[C_{A}+\phi_{1}\left(C_{h}+\theta C_{d}\right)+\phi_{2}\left(C_{p}+C\right)+\phi_{4} C_{r} k a+\phi_{30} C_{p} I_{c 2}-\phi_{28} P_{e}\right]}{\left[\phi_{5}\left(C_{h}+\theta C_{d}\right)+\phi_{11}\left(C_{p}+C\right)+\phi_{10} C_{r} k a+\phi_{31} C_{p} I_{c 2}-\phi_{29} p I_{e}\right]}$ and the inequality $\phi_{36}\left(T^{*}\right)^{2}+\phi_{37} T^{*}+\phi_{38}>0$ where all the values of $\phi_{\mathrm{i}}$ 's are given in Appendix 3.

Proof. For minimization of the total cost $Z_{3},\left.\frac{d Z_{3}}{d T}\right|_{T=T^{*}}=0$ and $\left.\frac{d^{2} Z_{3}}{d T^{2}}\right|_{T=T^{*}}>0$ must be satisfied.

Now,

$$
\begin{aligned}
Z_{3}= & \frac{C_{A}}{T}+\frac{\left(C_{h}+\theta C_{d}\right)}{T}\left\{\frac{a}{(m+\theta)^{2}}\left\{(k-1) e^{-(m+\theta) t_{1}}+e^{-(m+\theta)\left(t_{1}-T\right)}-k\right\}+\frac{a\left(k t_{1}-T\right)}{(m+\theta)}\right\}+\frac{\left(C_{p}+C\right) k a t_{1}}{T} \\
& -\frac{p I_{e}}{T}\left[t_{1}\left\{a t_{1}+\frac{m(k-1) a t_{1}}{(m+\theta)}+\frac{m(k-1) a}{(m+\theta)^{2}}\left(e^{-(m+\theta) t_{1}}-1\right)\right\}+T\left\{a\left(S-t_{1}\right)+\frac{m a}{(m+\theta)^{2}}\left(e^{-(m+\theta)\left(t_{1}-T\right)}-e^{-(m+\theta)(S-T)}\right)\right.\right. \\
& \left.-\frac{m a\left(S-t_{1}\right)}{(m+\theta)}\right\}-\left\{\frac{a t_{1}^{2}}{2}+\frac{m(k-1) a t_{1}^{2}}{2(m+\theta)}+\frac{m(k-1) a t_{1} e^{-(m+\theta) t_{1}}}{(m+\theta)^{2}}+\frac{m(k-1) a}{(m+\theta)^{3}}\left(e^{-(m+\theta) t_{1}}-1\right)\right\}-\left\{\frac{a\left(S^{2}-t_{1}^{2}\right)}{2}\right. \\
& \left.\left.+\frac{m a}{(m+\theta)^{2}}\left(t_{1} e^{-(m+\theta)\left(t_{1}-T\right)}-S e^{-(m+\theta)(S-T)}\right)+\frac{m a}{(m+\theta)^{3}}\left(e^{-(m+\theta)\left(t_{1}-T\right)}-e^{-(m+\theta)(S-T)}\right)-\frac{m a\left(S^{2}-t_{1}^{2}\right)}{2(m+\theta)}\right\}\right] \\
& +\frac{C_{r} k a}{T}\left(1-e^{\left.-\left(\frac{\alpha}{\beta+1}\right) t^{\beta}\right)^{\beta+1}}\right)+\frac{I_{c 2} C_{P} a}{T(m+\theta)}\left\{S-T-\frac{1}{(m+\theta)}+\frac{e^{-(m+\theta)(S-T)}}{(m+\theta)}\right\} .
\end{aligned}
$$

Differentiating the above expression with respect to $\mathrm{T}$, we get

$$
\begin{aligned}
\frac{d Z_{3}}{d T}= & \frac{\phi_{5}\left(C_{h}+\theta C_{d}\right)}{T}-\frac{\phi_{1}\left(C_{h}+\theta C_{d}\right)}{T^{2}}-\frac{C_{A}}{T^{2}}-\frac{\phi_{2}\left(C_{p}+C\right)}{T^{2}}+\frac{\phi_{11}\left(C_{p}+C\right)}{T}+\frac{\phi_{28} p I_{e}}{T^{2}}-\frac{\phi_{29} p I_{e}}{T}-\frac{\phi_{4} C_{r} k a}{T^{2}} \\
& +\frac{\phi_{10} C_{r} k a}{T}-\frac{\phi_{30} C_{p} I_{c 2}}{T^{2}}+\frac{\phi_{31} C_{p} I_{c 2}}{T} .
\end{aligned}
$$

and again, differentiating the above expression with respect to $\mathrm{T}$, we have

$$
\begin{aligned}
\frac{d^{2} Z_{3}}{d T^{2}}= & \frac{2 C_{A}}{T^{3}}+\frac{2 \phi_{1}\left(C_{h}+\theta C_{d}\right)}{T^{3}}-\frac{2 \phi_{5}\left(C_{h}+\theta C_{d}\right)}{T^{2}}+\frac{\phi_{12}\left(C_{h}+\theta C_{d}\right)}{T}+\frac{2 \phi_{2}\left(C_{p}+C\right)}{T^{3}}-\frac{2 \phi_{11}\left(C_{p}+C\right)}{T^{2}}+\frac{\phi_{13}\left(C_{p}+C\right)}{T} \\
& -\frac{2 \phi_{28} p I_{e}}{T^{3}}+\frac{2 \phi_{29} p I_{e}}{T^{2}}-\frac{\phi_{34} p I_{e}}{T}+\frac{2 \phi_{4} C_{r} k a}{T^{3}}-\frac{2 \phi_{10} C_{r} k a}{T^{2}}+\frac{\phi_{15} C_{r} k a}{T}+\frac{2 \phi_{30} C_{p} I_{c 2}}{T^{3}}-\frac{2 \phi_{31} C_{p} I_{c 1}}{T^{2}}+\frac{\phi_{35} C_{p} I_{c 2}}{T}
\end{aligned}
$$


For minimum of $Z_{3},\left.\frac{d Z_{3}}{d T}\right|_{T=T^{*}}=0$ and $\left.\frac{d^{2} Z_{3}}{d T^{2}}\right|_{T=T^{*}}>0$ imply that

$\left[\frac{\phi_{s}\left(C_{h}+\theta C_{d}\right)}{T}+\frac{\phi_{1}\left(C_{p}+C\right)}{T}+\frac{\phi_{28} p I_{e}}{T^{2}}+\frac{\phi_{10} C_{r} k a}{T}+\frac{\phi_{31} C_{p} I_{c 2}}{T}\right]_{T=T^{*}}=\left[\frac{C_{A}}{T^{2}}+\frac{\phi\left(C_{h}+\theta C_{d}\right)}{T^{2}}+\frac{\phi_{2}\left(C_{p}+C\right)}{T^{2}}+\frac{\phi_{29} p I_{e}}{T}+\frac{\phi_{4} C_{r} k a}{T^{2}}+\frac{\phi_{30} C_{p} I_{c 2}}{T^{2}}\right]_{T=T^{*}}$,

and

$$
\begin{aligned}
& {\left[\frac{2 C_{A}}{T^{3}}+\frac{2 \phi_{1}\left(C_{h}+\theta C_{d}\right)}{T^{3}}+\frac{\phi_{12}\left(C_{h}+\theta C_{d}\right)}{T}+\frac{2 \phi_{2}\left(C_{p}+C\right)}{T^{3}}+\frac{\phi_{13}\left(C_{p}+C\right)}{T}+\frac{2 \phi_{29} p I_{e}}{T^{2}}+\frac{2 \phi_{4} C_{r} k a}{T^{3}}+\frac{\phi_{15} C_{r} k a}{T}+\frac{2 \phi_{30} C_{p} I_{c 2}}{T^{3}}+\frac{\phi_{35} C_{p} I_{c 2}}{T}\right]_{T=T^{*}}} \\
& >\left[\frac{2 \phi_{5}\left(C_{h}+\theta C_{d}\right)}{T^{2}}+\frac{2 \phi_{11}\left(C_{p}+C\right)}{T^{2}}+\frac{2 \phi_{28} p I_{e}}{T^{3}}+\frac{\phi_{34} p I_{e}}{T}+\frac{2 \phi_{10} C_{r} k a}{T^{2}}+\frac{2 \phi_{31} C_{p} I_{c 1}}{T^{2}}\right]_{T=T^{*}} .
\end{aligned}
$$

After some simplification, we get

$$
T^{*}=\frac{\left[C_{A}+\phi_{1}\left(C_{h}+\theta C_{d}\right)+\phi_{2}\left(C_{p}+C\right)+\phi_{4} C_{r} k a+\phi_{30} C_{p} I_{c 2}-\phi_{28} p I_{e}\right]}{\left[\phi_{5}\left(C_{h}+\theta C_{d}\right)+\phi_{11}\left(C_{p}+C\right)+\phi_{10} C_{r} k a+\phi_{31} C_{p} I_{c 2}-\phi_{29} p I_{e}\right]} \text { and } \phi_{36}\left(T^{*}\right)^{2}+\phi_{37} T^{*}+\phi_{38}>0 \text {. }
$$

\section{Algorithm}

Step 1: Determine $\mathrm{T}_{1}{ }^{*}$ from equation (22), if $\mathrm{T}_{1}{ }^{*} \leq \mathrm{R}$ then evaluate $\mathrm{Z}_{1}\left(\mathrm{~T}_{1}{ }^{*}\right)$ from (19). Otherwise go to step 2.

Step 2: Determine $\mathrm{T}_{2}{ }^{*}$ from equation (23), if $\mathrm{R} \leq \mathrm{T}_{1}{ }^{*} \leq \mathrm{S}$ then evaluate $\mathrm{Z}_{2}\left(\mathrm{~T}_{2}{ }^{*}\right)$ from (20). Otherwise go to step 3. Step 3: Determine $\mathrm{T}_{3}{ }^{*}$ from equation (24), if $\mathrm{T}_{1}{ }^{*} \geq \mathrm{S}$ then evaluate $\mathrm{Z}_{3}\left(\mathrm{~T}_{3}{ }^{*}\right)$ from (21). Otherwise go to step 4. Step 4: Find out $\mathrm{TC}=\min \left\{\mathrm{Z}_{1}\left(\mathrm{~T}_{1}{ }^{*}\right), \mathrm{Z}_{2}\left(\mathrm{~T}_{2}{ }^{*}\right), \mathrm{Z}_{3}\left(\mathrm{~T}_{3}{ }^{*}\right)\right\}$.

\section{Numerical Examples}

All calculations are executed with the help of the software Mathematica 8.0, from where we get the optimal value. To illustrate the proposed model two examples are presented here in which $Z_{1}$ and $Z_{3}$ are the optimal solution.

Example 1. We consider the following parameter values on the basis of the previous study:

$\mathrm{C}_{\mathrm{A}}=\$ 180$ /order, $\mathrm{p}=\$ 20$ /unit, $\mathrm{a}=15, \mathrm{~m}=0.5, \mathrm{k}=2, \theta=0.1, \mathrm{C}=2, \mathrm{C}_{\mathrm{d}}=15 /$ unit, $\mathrm{R}=1.5$ years, $\mathrm{I}_{\mathrm{e}}=$ $\$ 0.15 /$ year, $\mathrm{I}_{\mathrm{c} 1}=\$ 0.18 /$ year, $\mathrm{I}_{\mathrm{c} 2}=\$ 0.20 /$ year, $\mathrm{S}=1.74$ years, $\mathrm{C}_{\mathrm{h}}=\$ 14 /$ unit/year, $\mathrm{C}_{\mathrm{p}}=\$ 10 /$ unit, $\alpha=$ $0.010, \beta=0.053, \mathrm{C}_{\mathrm{r}}=\$ 1.5 /$ item. Then the optimal solutions are:

In, case (1): $\left\{\mathrm{T}_{1}{ }^{*}=1.2529, \mathrm{Z}_{1}\left(\mathrm{~T}_{1}{ }^{*}\right)=398.759\right\}$, case $(2):\left\{\mathrm{T}_{2}{ }^{*}=1.7157, \mathrm{Z}_{2}\left(\mathrm{~T}_{2}{ }^{*}\right)=401.442\right\}$, case (3): $\left\{\mathrm{T}_{3}{ }^{*}=1.76178, \mathrm{Z}_{3}\left(\mathrm{~T}_{3}{ }^{*}\right)=400.971\right\}$. Among the above optimal solutions, the better optimal solution $\mathrm{TC}=\min \left\{\mathrm{Z}_{1}\left(\mathrm{~T}_{1}{ }^{*}\right), \mathrm{Z}_{2}\left(\mathrm{~T}_{2}{ }^{*}\right), \mathrm{Z}_{3}\left(\mathrm{~T}_{3}{ }^{*}\right)\right\}=398.759, \mathrm{~T}^{*}=1.2529$. From the numerical example, Figs. 4-6 show the convexity of the cost function.

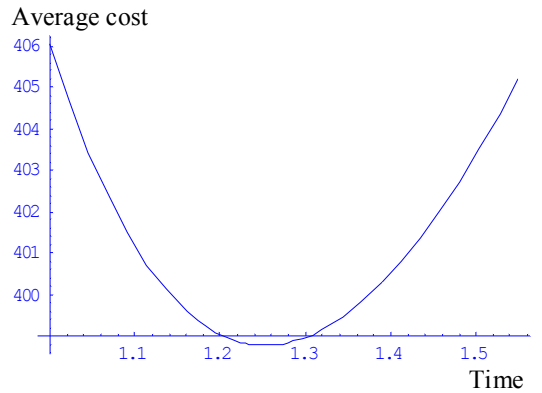

Fig. 4. Case 1: average cost versus cycle length

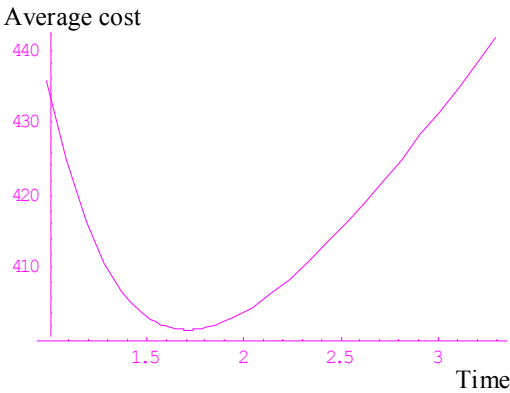

Fig. 5. Case 2: average cost versus cycle length

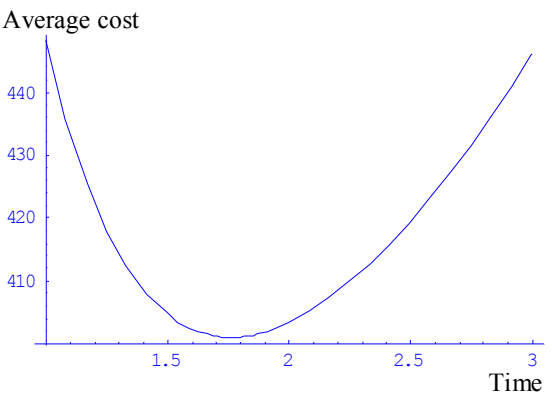

Fig. 6. Case 3: average cost versus cycle length 
Example 2. We consider the following parameter values on the basis of the previous study:

$\mathrm{C}_{\mathrm{A}}=\$ 350$ /order, $\mathrm{p}=\$ 20$ /unit, $\mathrm{a}=15, \mathrm{~m}=0.5, \mathrm{k}=2, \theta=0.1, \mathrm{C}=2, \mathrm{C}_{\mathrm{d}}=15 /$ unit, $\mathrm{R}=2.1$ years, $\mathrm{I}_{\mathrm{e}}=$ $\$ 0.15 /$ year, $\mathrm{I}_{\mathrm{c} 1}=\$ 0.18 /$ year, $\mathrm{I}_{\mathrm{c} 2}=\$ 0.20 /$ year, $\mathrm{S}=2.75$ years, $\mathrm{C}_{\mathrm{h}}=\$ 14 /$ unit/year, $\mathrm{C}_{\mathrm{p}}=\$ 10 /$ unit, $\alpha=$ $0.10, \beta=0.53, \mathrm{C}_{\mathrm{r}}=\$ 1.5 /$ item. Then the optimal solutions are:

For, case (1): $\left\{\mathrm{T}_{1}{ }^{*}=1.77939, \mathrm{Z}_{1}\left(\mathrm{~T}_{1}{ }^{*}\right)=480.611\right\}$, case $(2):\left\{\mathrm{T}_{2}{ }^{*}=2.66707, \mathrm{Z}_{2}\left(\mathrm{~T}_{2}{ }^{*}\right)=479.241\right\}$, case (3): \{ $\left.\mathrm{T}_{3}{ }^{*}=2.86137, \mathrm{Z}_{3}\left(\mathrm{~T}_{3}{ }^{*}\right)=476.712\right\}$. Among the above optimal solutions, the better optimal solution $\mathrm{TC}=\min \left\{\mathrm{Z}_{1}\left(\mathrm{~T}_{1}{ }^{*}\right), \mathrm{Z}_{2}\left(\mathrm{~T}_{2}{ }^{*}\right), \mathrm{Z}_{3}\left(\mathrm{~T}_{3}{ }^{*}\right)\right\}=476.712, \mathrm{~T}^{*}=2.86137$. From the numerical example, Figs. 7-9 show the convexity of the cost function.

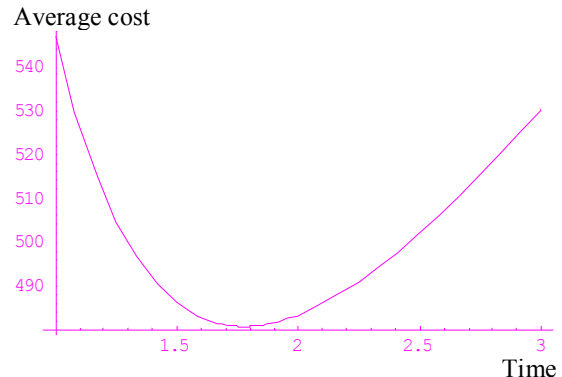

Fig. 7. Case 1: average cost versus cycle length

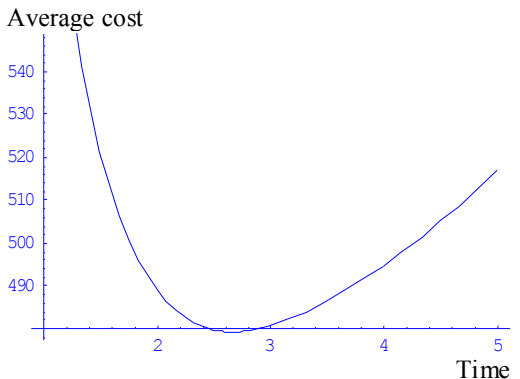

Fig. 8. Case 2: average cost versus cycle length

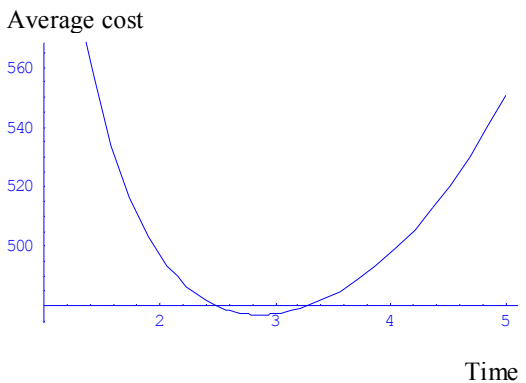

Fig. 9. Case 3: average cost versus cycle length

\section{Sensitivity Analysis}

Sensitivity analysis of the numerical example (1) and (2) are presented in Table 1 and 2 respectively as follows.

Table 1

(For example (1)) The effect of changing the parameter $(i)$ while keeping all other parameters unchanged

\begin{tabular}{|c|c|c|c|c|c|c|c|c|c|}
\hline Parameter (i) & $\%$ Change & $\mathrm{T}_{1}{ }^{*}$ & $\mathrm{Z}_{1}\left(\mathrm{~T}_{1}^{*}\right)$ & $\mathrm{T}_{2}^{*}$ & $\mathrm{Z}_{2}\left(\mathrm{~T}_{2}^{*}\right)$ & $\mathrm{T}_{3}{ }^{*}$ & $\mathrm{Z}_{3}\left(\mathrm{~T}_{3}{ }^{*}\right)$ & Optimal Solution & $\mathrm{TC}$ \\
\hline \multirow[t]{4}{*}{$\mathrm{C}_{\mathrm{p}}$} & -20 & 1.27669 & 363.199 & 1.78904 & 363.798 & 1.80790 & 363.295 & $\mathrm{Z}_{1}\left(\mathrm{~T}_{1}{ }^{*}\right)$ & 363.199 \\
\hline & -10 & 1.26464 & 380.991 & 1.75041 & 382.663 & 1.78406 & 382.157 & $\mathrm{Z}_{1}\left(\mathrm{~T}_{1}^{*}\right)$ & 380.991 \\
\hline & +10 & 1.24146 & 416.502 & 1.68424 & 420.145 & 1.74091 & 419.743 & $\mathrm{Z}_{1}\left(\mathrm{~T}_{1}{ }^{*}\right)$ & 416.502 \\
\hline & +20 & 1.23031 & 434.221 & 1.65554 & 438.778 & 1.72130 & 438.474 & $\mathrm{Z}_{1}\left(\mathrm{~T}_{1}{ }^{*}\right)$ & 434.221 \\
\hline \multirow{3}{*}{$\mathrm{C}_{\mathrm{A}}$} & -10 & 1.18809 & 384.010 & 1.62765 & 390.674 & 1.69824 & 390.567 & $\mathrm{Z}_{1}\left(\mathrm{~T}_{1}{ }^{*}\right)$ & 384.010 \\
\hline & +10 & 1.31476 & 412.780 & 1.80141 & 411.679 & 1.82282 & 411.014 & $\mathrm{Z}_{3}\left(\mathrm{~T}_{1}{ }^{\prime}\right)$ & 411.014 \\
\hline & +20 & 1.37410 & 426.168 & 1.88513 & 421.445 & 1.88157 & 420.732 & $\mathrm{Z}_{3}\left(\mathrm{~T}_{1}{ }^{*}\right)$ & 420.732 \\
\hline \multirow[t]{4}{*}{$\mathrm{p}$} & -20 & 1.29398 & 404.494 & 1.66931 & 406.189 & 1.72479 & 405.896 & $\mathrm{Z}_{3}\left(\mathrm{~T}_{1}{ }^{*}\right)$ & 404.494 \\
\hline & -10 & 1.27285 & 401.663 & 1.69182 & 403.836 & 1.74342 & 403.450 & $\mathrm{Z}_{1}\left(\mathrm{~T}_{1}{ }^{*}\right)$ & 401.663 \\
\hline & +10 & 1.23402 & 395.786 & 1.74109 & 399.006 & 1.77985 & 398.462 & $\mathrm{Z}_{1}\left(\mathrm{~T}_{1}{ }^{*}\right)$ & 395.786 \\
\hline & +20 & 1.21611 & 392.750 & 1.76823 & 396.525 & 1.79762 & 395.923 & $\mathrm{Z}_{1}\left(\mathrm{~T}_{1}{ }^{*}\right)$ & 392.750 \\
\hline \multirow[t]{4}{*}{$\mathrm{a}$} & -20 & 1.40293 & 346.120 & 1.92633 & 340.934 & 1.91014 & 340.384 & $\mathrm{Z}_{3}\left(\mathrm{~T}_{1}^{*}\right)$ & 340.384 \\
\hline & -10 & 1.32147 & 372.867 & 1.81080 & 371.508 & 1.82946 & 370.898 & $\mathrm{Z}_{3}\left(\mathrm{~T}_{1}{ }^{*}\right)$ & 370.898 \\
\hline & +10 & 1.19412 & 423.923 & 1.63576 & 430.844 & 1.70413 & 430.682 & $\mathrm{Z}_{1}\left(\mathrm{~T}_{1}{ }^{*}\right)$ & 423.923 \\
\hline & +20 & 1.14299 & 448.458 & 1.56744 & 459.794 & 1.65439 & 460.090 & $\mathrm{Z}_{1}\left(\mathrm{~T}_{1}^{*}\right)$ & 448.458 \\
\hline \multirow[t]{4}{*}{$\mathrm{m}$} & -20 & 1.26871 & 394.754 & 1.71542 & 396.728 & 1.75925 & 396.267 & $\mathrm{Z}_{1}\left(\mathrm{~T}_{1}{ }^{*}\right)$ & 394.754 \\
\hline & -10 & 1.26044 & 396.787 & 1.71438 & 399.144 & 1.75957 & 398.681 & $\mathrm{Z}_{1}\left(\mathrm{~T}_{1}{ }^{*}\right)$ & 396.787 \\
\hline & +10 & 1.24604 & 400.671 & 1.71950 & 403.624 & 1.76595 & 403.136 & $\mathrm{Z}_{1}\left(\mathrm{~T}_{1}{ }^{*}\right)$ & 400.671 \\
\hline & +20 & 1.23984 & 402.524 & 1.72598 & 405.686 & 1.77218 & 405.173 & $Z_{1}\left(T_{1}^{*}\right)$ & 402.524 \\
\hline \multirow{3}{*}{$\mathrm{k}$} & -10 & 1.33769 & 384.015 & 1.95273 & 379.477 & 1.92372 & 378.815 & $\mathrm{Z}_{3}\left(\mathrm{~T}_{1}^{*}\right)$ & 378.815 \\
\hline & +10 & 1.19275 & 410.296 & 1.57058 & 418.069 & 1.64509 & 418.345 & $\mathrm{Z}_{1}\left(\mathrm{~T}_{1}{ }^{*}\right)$ & 410.296 \\
\hline & +20 & 1.14799 & 419.561 & 1.47257 & 431.129 & 1.55900 & 432.275 & $\mathrm{Z}_{1}\left(\mathrm{~T}_{1}{ }^{*}\right)$ & 419.561 \\
\hline \multirow[t]{4}{*}{$\theta$} & -20 & 1.26114 & 396.444 & 1.73148 & 398.526 & 1.77314 & 398.008 & $\mathrm{Z}_{1}\left(\mathrm{~T}_{1}{ }^{*}\right)$ & 396.444 \\
\hline & -10 & 1.25699 & 397.605 & 1.72349 & 399.990 & 1.76740 & 399.495 & $\mathrm{Z}_{1}\left(\mathrm{~T}_{1}{ }^{*}\right)$ & 397.605 \\
\hline & +10 & 1.24887 & 399.907 & 1.70808 & 402.883 & 1.75627 & 402.437 & $\mathrm{Z}_{1}\left(\mathrm{~T}_{1}{ }^{*}\right)$ & 399.907 \\
\hline & +20 & 1.24490 & 401.049 & 1.70063 & 404.313 & 1.75088 & 403.891 & $\mathrm{Z}_{1}\left(\mathrm{~T}_{1}{ }^{*}\right)$ & 401.049 \\
\hline \multirow[t]{4}{*}{$\mathrm{C}_{\mathrm{d}}$} & -20 & 1.25873 & 397.378 & 1.72995 & 399.585 & 1.77243 & 399.070 & $\mathrm{Z}_{1}\left(\mathrm{~T}_{1}{ }^{*}\right)$ & 397.378 \\
\hline & -10 & 1.25580 & 398.069 & 1.72278 & 400.515 & 1.76709 & 400.022 & $\mathrm{Z}_{1}\left(\mathrm{~T}_{1}{ }^{*}\right)$ & 398.069 \\
\hline & +10 & 1.25001 & 399.447 & 1.70870 & 402.366 & 1.75651 & 401.918 & $\mathrm{Z}_{1}\left(\mathrm{~T}_{1}{ }^{*}\right)$ & 399.447 \\
\hline & +20 & 1.24714 & 400.133 & 1.70179 & 403.286 & 1.75128 & 402.862 & $\mathrm{Z}_{1}\left(\mathrm{~T}_{1}{ }^{*}\right)$ & 400.133 \\
\hline \multirow[t]{4}{*}{$\mathrm{C}_{\mathrm{r}}$} & -20 & 1.25294 & 398.709 & 1.71580 & 401.389 & 1.76186 & 400.918 & $\mathrm{Z}_{1}\left(\mathrm{~T}_{1}^{*}\right)$ & 398.709 \\
\hline & -10 & 1.25292 & 398.734 & 1.71575 & 401.416 & 1.76182 & 400.945 & $\mathrm{Z}_{1}\left(\mathrm{~T}_{1}{ }^{*}\right)$ & 398.734 \\
\hline & +10 & 1.25287 & 398.784 & 1.71564 & 401.469 & 1.76174 & 400.998 & $\mathrm{Z}_{1}\left(\mathrm{~T}_{1}{ }^{*}\right)$ & 398.784 \\
\hline & +20 & 1.25285 & 398.808 & 1.71559 & 41.4960 & 1.76170 & 401.025 & $\mathrm{Z}_{1}\left(\mathrm{~T}_{1}{ }^{*}\right)$ & 398.808 \\
\hline \multirow[t]{4}{*}{$\mathrm{C}$} & -20 & 1.25756 & 391.655 & 1.72707 & 393.958 & 1.77029 & 393.451 & $\mathrm{Z}_{1}\left(\mathrm{~T}_{1}{ }^{*}\right)$ & 391.655 \\
\hline & -10 & 1.25522 & 395.207 & 1.72136 & 397.701 & 1.76602 & 397.212 & $\mathrm{Z}_{1}\left(\mathrm{~T}_{1}{ }^{*}\right)$ & 395.207 \\
\hline & +10 & 1.25058 & 402.309 & 1.71009 & 405.181 & 1.75756 & 404.729 & $\mathrm{Z}_{1}\left(\mathrm{~T}_{1}{ }^{*}\right)$ & 402.309 \\
\hline & +20 & 1.24829 & 405.859 & 1.70454 & 408.918 & 1.75336 & 408.485 & $\mathrm{Z}_{1}\left(\mathrm{~T}_{1}{ }^{*}\right)$ & 405.859 \\
\hline $\mathrm{C}_{\mathrm{h}}$ & +20 & 1.20191 & 411.36 & 1.59766 & 418.145 & 1.66930 & 418.231 & $\mathrm{Z}_{1}\left(\mathrm{~T}_{1}{ }^{*}\right)$ & 411.360 \\
\hline
\end{tabular}


Table 2

(For example (2)) The effect of changing the parameter $(i)$ while keeping all other parameters unchanged

\begin{tabular}{|c|c|c|c|c|c|c|c|c|c|}
\hline Parameter (i) & $\%$ Change & $\mathrm{T}_{1}{ }^{*}$ & $\mathrm{Z}_{1}\left(\mathrm{~T}_{1}^{*}\right)$ & $\mathrm{T}_{2}{ }^{*}$ & $\mathrm{Z}_{2}\left(\mathrm{~T}_{2}^{*}\right)$ & $\mathrm{T}_{3}{ }^{*}$ & $\mathrm{Z}_{3}\left(\mathrm{~T}_{3}{ }^{*}\right)$ & Optimal Solution & $\mathrm{TC}$ \\
\hline \multirow[t]{4}{*}{$\mathrm{C}_{\mathrm{p}}$} & -20 & 1.81323 & 442.892 & 2.85506 & 437.557 & 2.95676 & 434.985 & $\mathrm{Z}_{3}\left(\mathrm{~T}_{3}^{*}\right)$ & 434.985 \\
\hline & -10 & 1.79610 & 461.768 & 2.75217 & 458.509 & 2.90722 & 455.891 & $\mathrm{Z}_{3}\left(\mathrm{~T}_{3}{ }^{*}\right)$ & 455.891 \\
\hline & +10 & 1.76310 & 499.422 & 2.59462 & 499.761 & 2.81884 & 497.456 & $\mathrm{Z}_{3}\left(\mathrm{~T}_{3}{ }^{*}\right)$ & 497.456 \\
\hline & +20 & 1.74721 & 518.201 & 2.53165 & 520.188 & 2.77928 & 518.130 & $\mathrm{Z}_{3}\left(\mathrm{~T}_{3}{ }^{*}\right)$ & 518.130 \\
\hline \multirow[t]{4}{*}{$\mathrm{C}_{\mathrm{A}}$} & -20 & 1.58531 & 438.994 & 2.33311 & 451.201 & 2.63309 & 451.236 & $\mathrm{Z}_{1}\left(\mathrm{~T}_{1}{ }^{*}\right)$ & 438.994 \\
\hline & -10 & 1.68462 & 460.402 & 2.50028 & 465.689 & 2.75028 & 464.239 & $\mathrm{Z}_{1}\left(\mathrm{~T}_{1}^{*}\right)$ & 460.402 \\
\hline & +10 & 1.87037 & 499.791 & 2.83369 & 491.970 & 2.96645 & 488.723 & $\mathrm{Z}_{3}\left(\mathrm{~T}_{3}{ }^{*}\right)$ & 488.723 \\
\hline & +20 & 1.95811 & 518.076 & 2.99996 & 503.972 & 3.06568 & 500.326 & $\mathrm{Z}_{3}\left(\mathrm{~T}_{3}{ }^{*}\right)$ & 500.326 \\
\hline \multirow[t]{3}{*}{$\mathrm{p}$} & -20 & 1.84287 & 488.940 & 2.53810 & 487.481 & 2.75589 & 485.854 & $\mathrm{Z}_{3}\left(\mathrm{~T}_{3}{ }^{*}\right)$ & 485.854 \\
\hline & +10 & 1.75060 & 476.285 & 2.74591 & 474.893 & 2.91322 & 471.977 & $\mathrm{Z}_{3}\left(\mathrm{~T}_{3}{ }^{*}\right)$ & 471.977 \\
\hline & +20 & 1.72349 & 471.860 & 2.83880 & 470.358 & 2.96407 & 467.139 & $\mathrm{Z}_{3}\left(\mathrm{~T}_{3}{ }^{*}\right)$ & 467.139 \\
\hline \multirow[t]{4}{*}{$\mathrm{a}$} & -20 & 2.00092 & 421.533 & 3.08277 & 407.781 & 3.11316 & 404.793 & $\mathrm{Z}_{3}\left(\mathrm{~T}_{3}{ }^{*}\right)$ & 404.793 \\
\hline & -10 & 1.88027 & 451.679 & 2.85219 & 444.004 & 2.97776 & 441.028 & $\mathrm{Z}_{3}\left(\mathrm{~T}_{3}{ }^{*}\right)$ & 441.028 \\
\hline & +10 & 1.69341 & 508.515 & 2.51545 & 513.654 & 2.76063 & 511.933 & $\mathrm{Z}_{1}\left(\mathrm{~T}_{1}^{*}\right)$ & 508.515 \\
\hline & +20 & 1.61897 & 535.531 & 2.38891 & 547.372 & 2.67284 & 546.76 & $\mathrm{Z}_{1}\left(\mathrm{~T}_{1}{ }^{*}\right)$ & 535.531 \\
\hline \multirow[t]{3}{*}{$\mathrm{m}$} & -20 & 1.79006 & 476.407 & 2.56167 & 476.096 & 2.75873 & 474.394 & $\mathrm{Z}_{3}\left(\mathrm{~T}_{3}{ }^{*}\right)$ & 474.394 \\
\hline & -10 & 1.78397 & 478.573 & 2.60677 & 477.862 & 2.80456 & 475.777 & $\mathrm{Z}_{3}\left(\mathrm{~T}_{3}{ }^{*}\right)$ & 475.777 \\
\hline & +10 & 1.77627 & 482.525 & 2.74735 & 480.188 & 2.92970 & 477.175 & $\mathrm{Z}_{3}\left(\mathrm{~T}_{3}{ }^{*}\right)$ & 477.175 \\
\hline \multirow{3}{*}{$\mathrm{k}$} & -10 & 1.91876 & 458.746 & 3.25081 & 442.920 & 3.19904 & 439.201 & $\mathrm{Z}_{3}\left(\mathrm{~T}_{3}{ }^{*}\right)$ & 439.201 \\
\hline & +10 & 1.68079 & 497.859 & 2.34213 & 506.344 & 2.58945 & 506.624 & $\mathrm{Z}_{1}\left(\mathrm{~T}_{1}{ }^{*}\right)$ & 497.859 \\
\hline & +20 & 1.60773 & 511.782 & 2.14123 & 527.412 & 2.38914 & 530.605 & $\mathrm{Z}_{1}\left(\mathrm{~T}_{1}^{*}\right)$ & 511.782 \\
\hline \multirow[t]{4}{*}{$\theta$} & -20 & 1.78812 & 477.607 & 2.67743 & 475.658 & 2.86441 & 473.100 & $\mathrm{Z}_{3}\left(\mathrm{~T}_{3}{ }^{*}\right)$ & 473.100 \\
\hline & -10 & 1.78371 & 479.114 & 2.67205 & 477.460 & 2.86273 & 474.917 & $\mathrm{Z}_{3}\left(\mathrm{~T}_{3}{ }^{*}\right)$ & 474.917 \\
\hline & +10 & 1.77517 & 482.098 & 2.66246 & 481.001 & 2.86033 & 478.484 & $\mathrm{Z}_{3}\left(\mathrm{~T}_{3}{ }^{*}\right)$ & 478.484 \\
\hline & +20 & 1.77103 & 483.575 & 2.65824 & 482.742 & 2.85960 & 480.235 & $\mathrm{Z}_{3}\left(\mathrm{~T}_{3}{ }^{*}\right)$ & 480.235 \\
\hline \multirow[t]{4}{*}{$\mathrm{C}_{\mathrm{d}}$} & -20 & 1.78769 & 478.694 & 2.69617 & 476.502 & 2.88205 & 473.816 & $\mathrm{Z}_{3}\left(\mathrm{~T}_{3}{ }^{*}\right)$ & 473.816 \\
\hline & -10 & 1.78353 & 479.653 & 2.68150 & 477.874 & 2.87169 & 475.266 & $\mathrm{Z}_{3}\left(\mathrm{~T}_{3}{ }^{*}\right)$ & 475.266 \\
\hline & +10 & 1.77528 & 481.567 & 2.65286 & 480.601 & 2.85111 & 478.154 & $\mathrm{Z}_{3}\left(\mathrm{~T}_{3}{ }^{*}\right)$ & 478.154 \\
\hline & +20 & 1.77120 & 482.520 & 2.63887 & 481.955 & 2.84091 & 479.591 & $\mathrm{Z}_{3}\left(\mathrm{~T}_{3}{ }^{*}\right)$ & 479.591 \\
\hline \multirow[t]{4}{*}{$\mathrm{C}_{\mathrm{r}}$} & -20 & 1.78072 & 480.234 & 2.67172 & 478.732 & 2.8647 & 476.178 & $\mathrm{Z}_{3}\left(\mathrm{~T}_{3}{ }^{*}\right)$ & 476.178 \\
\hline & -10 & 1.78005 & 480.423 & 2.66939 & 478.987 & 2.86304 & 476.445 & $\mathrm{Z}_{3}\left(\mathrm{~T}_{3}{ }^{*}\right)$ & 476.445 \\
\hline & +10 & 1.77873 & 480.799 & 2.66475 & 479.494 & 2.85971 & 476.979 & $\mathrm{Z}_{3}\left(\mathrm{~T}_{3}{ }^{*}\right)$ & 476.979 \\
\hline & +20 & 1.77807 & 480.988 & 2.66244 & 479.748 & 2.85805 & 477.246 & $\mathrm{Z}_{3}\left(\mathrm{~T}_{3}{ }^{\prime \prime}\right)$ & 477.246 \\
\hline \multirow[t]{4}{*}{$\mathrm{C}$} & -20 & 1.78602 & 473.078 & 2.69027 & 471.052 & 2.87790 & 468.397 & $\mathrm{Z}_{3}\left(\mathrm{~T}_{3}{ }^{*}\right)$ & 468.397 \\
\hline & -10 & 1.78270 & 476.845 & 2.6786 & 475.148 & 2.86962 & 472.556 & $\mathrm{Z}_{3}\left(\mathrm{~T}_{3}{ }^{*}\right)$ & 472.556 \\
\hline & +10 & 1.77610 & 484.376 & 2.65568 & 483.329 & 2.85316 & 480.866 & $\mathrm{Z}_{3}\left(\mathrm{~T}_{3}{ }^{*}\right)$ & 480.866 \\
\hline & +20 & 1.77283 & 488.139 & 2.64444 & 487.414 & 2.84498 & 485.016 & $\mathrm{Z}_{3}\left(\mathrm{~T}_{3}{ }^{*}\right)$ & 485.016 \\
\hline \multirow{2}{*}{$\mathrm{C}_{\mathrm{h}}$} & +10 & 1.74200 & 489.454 & 2.54265 & 491.709 & 2.76771 & 490.006 & $\mathrm{Z}_{1}\left(\mathrm{~T}_{1}{ }^{*}\right)$ & 489.454 \\
\hline & +20 & 1.70667 & 498.128 & 2.43429 & 503.71 & 2.67903 & 502.947 & $\mathrm{Z}_{1}\left(\mathrm{~T}_{1}{ }^{*}\right)$ & 498.128 \\
\hline
\end{tabular}

The behavior of the parameters changed with respect to the total average cost is shown graphically in Fig. 10 (for example (1)) and Fig. 11 (for example (2)) and some interesting results drawn from sensitivity analysis are given as follows.

(1) The total average cost increases as the purchasing cost $\left(C_{p}\right)$ increases, which is true in practical situation. As the purchasing cost per item increases, it is obvious to increase the optimal cost of the system.

(2) The total average cost of the system increases with an increase in ordering cost $\left(C_{A}\right)$, which is quite natural as the per order growth of ordering cost implies an increase in total average cost of the system.

(3) When the selling price increases the total average cost of the inventory system decreases. The fact is that due to the higher selling price retailer accumulates more revenue and earns more interest during the delay period.

(4) As the demand parameters $(a, m)$ increases the total average cost of the system increases. The motive is that more demand means more production consequently the total average cost increases.

(5) An increase in production parameter $(k)$ shows that the retailer produces more items therefore the holding cost and deterioration cost, etc. increases as a result the optimal cost of the system increases.

(6) The total average cost decreases as the deterioration rate $(\theta)$ and the deterioration $\operatorname{cost}\left(C_{d}\right)$ decreases which according to the real situation.

(7) An increase in rework cost per unit item indicates the growth of the total rework cost. To reduce the cost, the production of imperfect items will have to be reduced.

(8) When the production cost $(C)$ and the holding cost $\left(C_{h}\right)$ increases the total average cost of the system increases. The reason is that per unit increase in production and holding costs increases the total production and holding costs therefore the total average cost of the proposed model increases. 


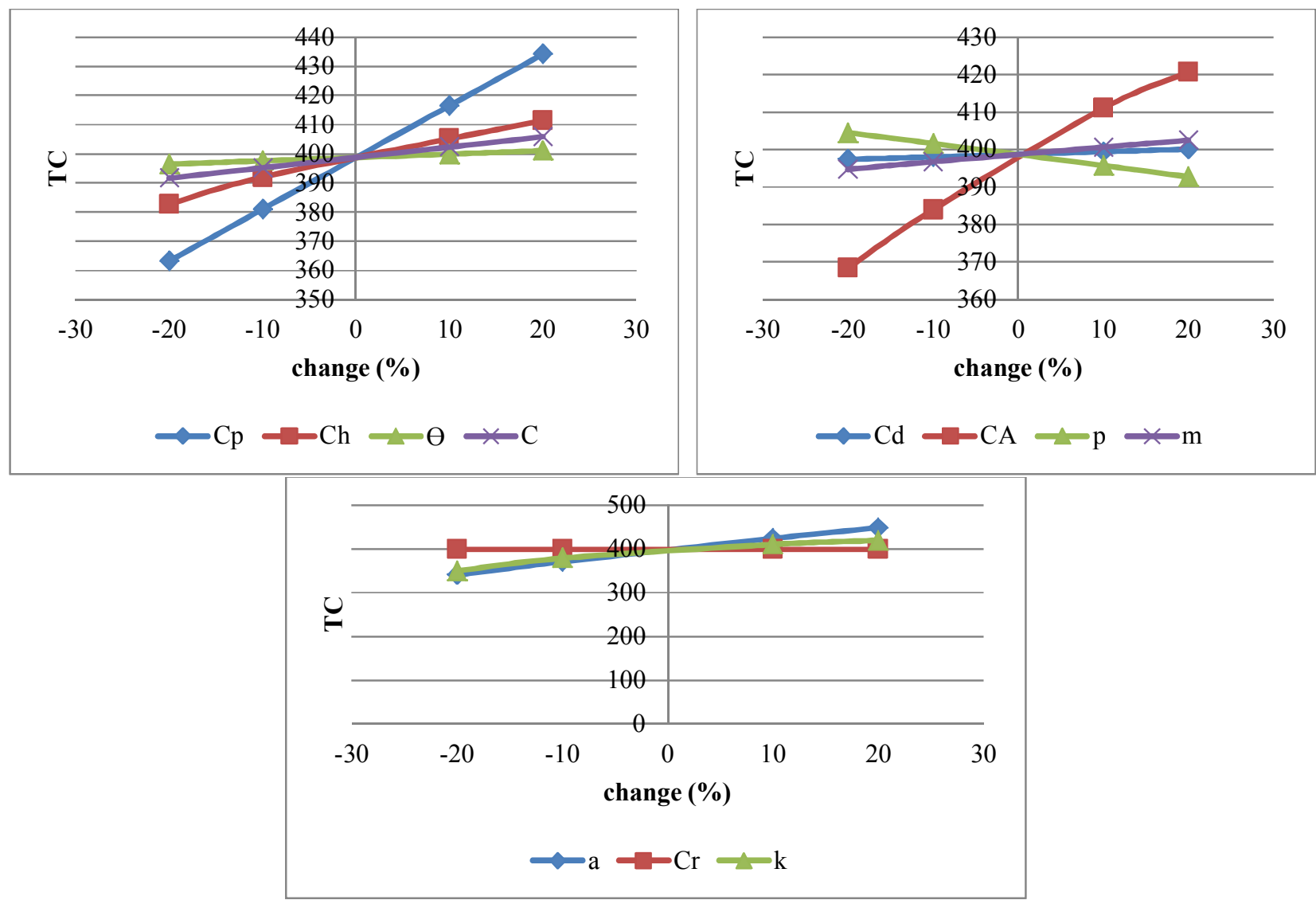

Fig. 10. (For example (1)) The effect of changing parameters on the optimal cost function

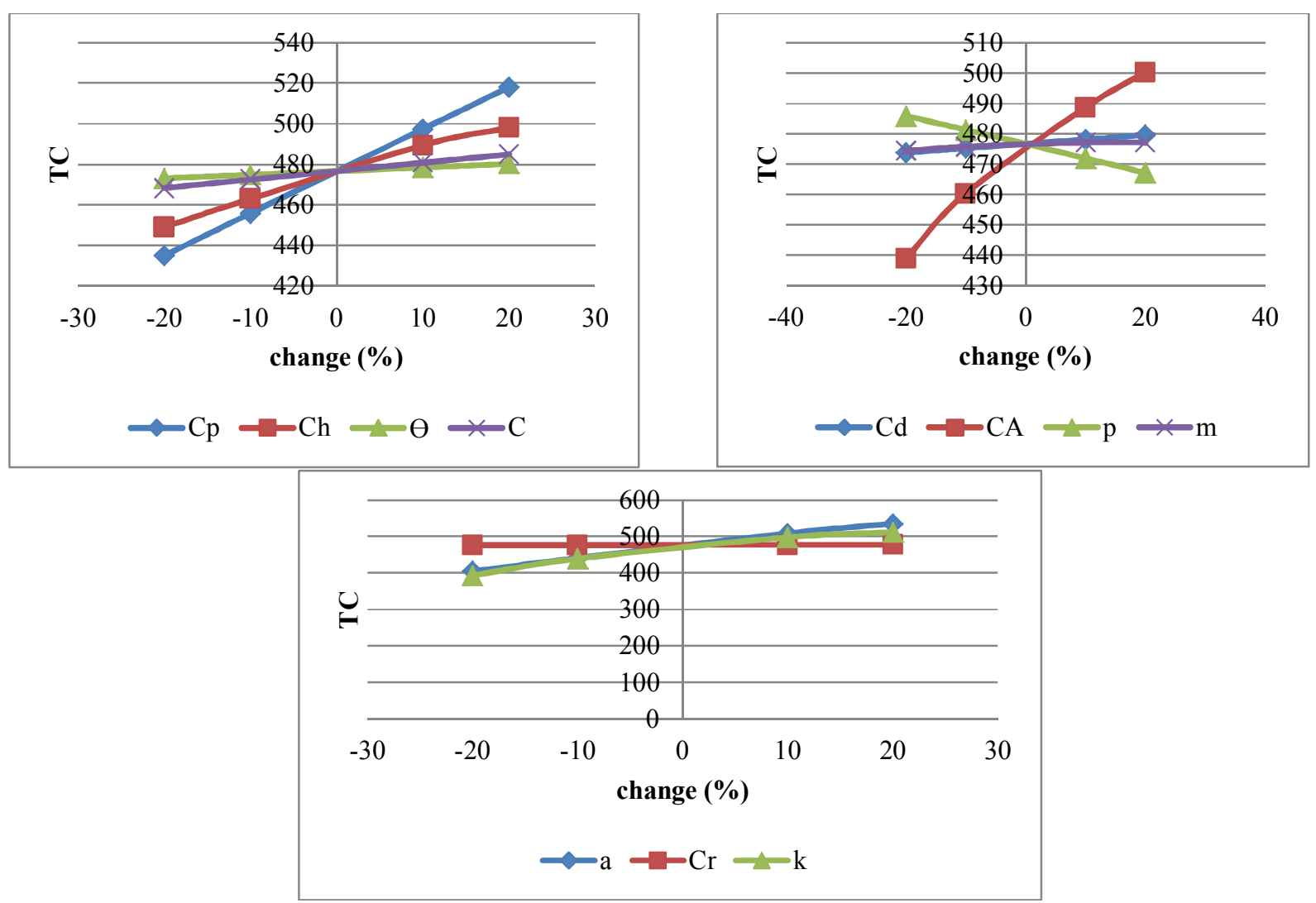

Fig. 11. (For example (2)) The effect of changing parameters on the optimal cost function 


\section{Conclusion}

In this research article, an inventory model for deteriorating items with stock dependent demand rate considering imperfect production and delay in payment scheme has been developed. In this model, two delay periods have been provided by the supplier to attract the retailer. During the delay period an interest was earned on accumulated revenue by the retailer selling his/her commodity. In most of the papers, the examiners have considered the production of the perfect items through different machinery systems. However, in practical situation, due to employment problems, machine breakdowns, the system produces imperfect quality items, which may rework at a cost to make it perfect. In this model, the production of the imperfect items follows Weibull distribution and the production rate depends on the demand factor. An algorithm to determine the optimal policy has also been presented. In addition, sensitivity analysis is performed to examine the effect of parameters. From sensitivity analysis it is observed that the model is enough stable with respect to the changes in system parameters. Further, the model may be generalized by considering shortages and $\mathrm{n}$ cycles in a finite planning horizon.

\section{Appendix 1.}

The values are given as follows:

$$
\begin{aligned}
& \phi_{1}=\frac{a e^{-(m+\theta) t_{1}}}{(m+\theta)^{2}}\left\{(k-1)+e^{(m+\theta) T}\right\}-\frac{a k}{(m+\theta)^{2}}+\frac{a\left(k t_{1}-T\right)}{(m+\theta)} \quad \phi_{2}=k a t_{1} \\
& \phi_{3}=\left[\left(R-T+t_{1}\right)\left\{a t_{1}+\frac{m(k-1) a t_{1}}{(m+\theta)}+\frac{m(k-1) a}{(m+\theta)^{2}}\left(e^{-(m+\theta) t_{1}}-1\right)\right\}+R\left\{a\left(T-t_{1}\right)+\frac{m a}{(m+\theta)^{2}}\left(e^{-(m+\theta)\left(t_{1}-T\right)}-1\right)-\frac{m a\left(T-t_{1}\right)}{(m+\theta)}\right\}\right. \\
& \left.-\left\{\frac{a T^{2}}{2}+\frac{m(k-1) a t_{1}^{2}}{2(m+\theta)}+\frac{m(k-1) a t_{1} e^{-(m+\theta) t_{1}}}{(m+\theta)^{2}}+\frac{m(k-1) a}{(m+\theta)^{3}}\left(e^{-(m+\theta) t_{1}}-1\right)+\frac{m a}{(m+\theta)^{2}}\left(t_{1} e^{-(m+\theta)\left(t_{1}-T\right)}-T\right)+\frac{m a}{(m+\theta)^{3}}\left(e^{-(m+\theta)\left(t_{1}-T\right)}-1\right)-\frac{m a\left(T^{2}-t_{1}^{2}\right)}{2(m+\theta)}\right\}\right] \\
& \phi_{4}=\left[1-e^{-\left(\frac{\alpha}{\beta+1}\right) t_{1}^{\beta+1}}\right], \phi_{5}=\frac{d \phi_{1}}{d T}=\frac{a(k-1)\left(1-e^{-(m+\theta) T}\right)}{(m+\theta)\left[1+(k-1) e^{-(m+\theta) T}\right]}, \\
& \phi_{6}=\frac{d \phi_{3}}{d T}=\left(R-T+t_{1}\right) \frac{d \phi_{7}}{d T}-\frac{(k-1) \phi_{7}}{\left[e^{(m+\theta) T}+(k-1)\right]}+R \frac{d \phi_{8}}{d T}-\frac{d \phi_{9}}{d T} \\
& \phi_{7}=\left\{a t_{1}+\frac{m(k-1) a t_{1}}{(m+\theta)}+\frac{m(k-1) a}{(m+\theta)^{2}}\left(e^{-(m+\theta) t_{1}}-1\right)\right\} \\
& \phi_{8}=\left\{a\left(T-t_{1}\right)+\frac{m a}{(m+\theta)^{2}}\left(e^{-(m+\theta)\left(t_{1}-T\right)}-1\right)-\frac{m a\left(T-t_{1}\right)}{(m+\theta)}\right\} \\
& \phi_{9}=\left\{\frac{a T^{2}}{2}+\frac{m(k-1) a t_{1}^{2}}{2(m+\theta)}+\frac{m(k-1) a t_{1} e^{-(m+\theta) t_{1}}}{(m+\theta)^{2}}+\frac{m(k-1) a}{(m+\theta)^{3}}\left(e^{-(m+\theta) t_{1}}-1\right)+\frac{m a}{(m+\theta)^{2}}\left(t_{1} e^{-(m+\theta)\left(t_{1}-T\right)}-T\right)\right. \\
& \left.+\frac{m a}{(m+\theta)^{3}}\left(e^{-(m+\theta)\left(t_{1}-T\right)}-1\right)-\frac{m a\left(T^{2}-t_{1}^{2}\right)}{2(m+\theta)}\right\} \\
& \frac{d \phi_{7}}{d T}=\frac{a}{\left[1+(k-1) e^{-(m+\theta) T}\right]}\left\{1+\frac{m(k-1)}{(m+\theta)}\left(1-e^{-(m+\theta) t_{1}}\right)\right\} \quad \frac{d \phi_{8}}{d T}=\frac{a(k-1)\left\{\theta+m e^{-(m+\theta)\left(t_{1}-T\right)}\right\}}{(m+\theta)\left[e^{(m+\theta) T}+(k-1)\right]} \\
& \frac{d \phi_{9}}{d T}=\frac{m a\left\{k t_{1}+(k-1) e^{-(m+\theta) t_{1}}+\frac{e^{-(m+\theta)\left(t_{1}-T\right)}}{(m+\theta)}\right\}}{(m+\theta)\left[1+(k-1) e^{-(m+\theta) T}\right]}+\left\{\frac{a \theta T}{(m+\theta)}-\frac{a m}{(m+\theta)^{2}}\right\}
\end{aligned}
$$




$$
\begin{aligned}
& \phi_{10}=\frac{d \phi_{4}}{d T}=\frac{\alpha t_{1}^{\beta} e^{-\left(\frac{\alpha}{\beta+1}\right) t_{1}^{\beta+1}}}{\left[1+(k-1) e^{-(m+\theta) T}\right]} \quad \phi_{11}=\frac{k a}{\left[1+(k-1) e^{-(m+\theta) T}\right]} \\
& \phi_{12}=\frac{d \phi_{5}}{d T}=\frac{k a(k-1) e^{-(m+\theta) T}}{\left[1+(k-1) e^{-(m+\theta) T}\right]^{2}} \quad \phi_{13}=\frac{d \phi_{11}}{d T}=\frac{k a(k-1)(m+\theta) e^{-(m+\theta) T}}{\left[1+(k-1) e^{-(m+\theta) T}\right]^{2}} \\
& \phi_{14}=\frac{d \phi_{6}}{d T}=\frac{(k-1)(m+\theta) e^{-(m+\theta) T}}{\left[1+(k-1) e^{-(m+\theta) T}\right]^{2}} \phi_{7}-\frac{2(k-1) e^{-(m+\theta) T}}{\left[1+(k-1) e^{-(m+\theta) T}\right]} \frac{d \phi_{7}}{d T}+\left(R-T+t_{1}\right) \frac{d^{2} \phi_{7}}{d T^{2}}+R \frac{d^{2} \phi_{8}}{d T^{2}}-\frac{d^{2} \phi_{9}}{d T^{2}} \\
& \frac{d^{2} \phi_{7}}{d T^{2}}=\frac{a(k-1)}{\left[1+(k-1) e^{-(m+\theta) T}\right]^{2}}\left[m e^{-(m+\theta) t_{1}}+e^{-(m+\theta) T}\left\{1+\frac{m(k-1)}{(m+\theta)}\left(1-e^{-(m+\theta) t_{1}}\right)\right\}\right] \\
& \frac{d^{2} \phi_{8}}{d T^{2}}=\frac{a(k-1)}{\left[e^{(m+\theta) T}+(k-1)\right]^{2}}\left[m(k-1) e^{-(m+\theta)\left(t_{1}-T\right)}-e^{(m+\theta) T}\left(\theta+m e^{-(m+\theta)\left(t_{1}-T\right)}\right)\right] \\
& \frac{d^{2} \phi_{9}}{d T^{2}}=\frac{m a(k-1) e^{-(m+\theta) T}\left\{(k-1) e^{-(m+\theta) t_{1}}+k t_{1}\right\}}{\left[1+(k-1) e^{-(m+\theta) T}\right]^{2}}+\frac{m a(k-1) e^{-(m+\theta) T}\left\{k+(k-1) e^{-(m+\theta) t_{1}}\right\}}{\left[1+(k-1) e^{-(m+\theta) T}\right]^{2}}+\frac{a \theta}{(m+\theta)} \\
& \phi_{15}=\frac{d \phi_{10}}{d T}=\frac{\alpha t_{1}^{\beta-1} e^{-\left(\frac{\alpha}{\beta+1}\right) t_{1}^{\beta+1}}}{\left[1+(k-1) e^{-(m+\theta) T}\right]^{2}}\left[(k-1)(m+\theta) t_{1} e^{-(m+\theta) T}+\beta-\alpha t_{1}^{\beta+1}\right] \\
& \phi_{16}=\left[\phi_{12}\left(C_{h}+\theta C_{d}\right)+\phi_{13}\left(C_{p}+C\right)+\phi_{15} C_{r} k a-\phi_{14} p I_{e}\right] \\
& \phi_{17}=2\left[\phi_{6} p I_{e}-\phi_{5}\left(C_{h}+\theta C_{d}\right)-\phi_{11}\left(C_{p}+C\right)-\phi_{10} C_{r} k a\right] \\
& \phi_{18}=2\left[C_{A}+\phi_{1}\left(C_{h}+\theta C_{d}\right)+\phi_{2}\left(C_{p}+C\right)+\phi_{4} C_{r} k a-\phi_{3} p I_{e}\right]
\end{aligned}
$$

\section{Appendix 2.}

The values are given as follows:

$$
\begin{aligned}
\phi_{19}= & {\left[t_{1}\left\{a t_{1}+\frac{m(k-1) a t_{1}}{(m+\theta)}+\frac{m(k-1) a}{(m+\theta)^{2}}\left(e^{-(m+\theta) t_{1}}-1\right)\right\}+T\left\{a\left(T-t_{1}\right)+\frac{m a}{(m+\theta)^{2}}\left(e^{-(m+\theta)\left(t_{1}-T\right)}-1\right)-\frac{m a\left(T-t_{1}\right)}{(m+\theta)}\right\}\right.} \\
& -\left\{\frac{a T^{2}}{2}+\frac{m(k-1) a t_{1}^{2}}{2(m+\theta)}+\frac{m(k-1) a t_{1} e^{-(m+\theta) t_{1}}}{(m+\theta)^{2}}+\frac{m(k-1) a}{(m+\theta)^{3}}\left(e^{-(m+\theta) t_{1}}-1\right)+\frac{m a}{(m+\theta)^{2}}\left(t_{1} e^{-(m+\theta)\left(t_{1}-T\right)}-T\right)\right. \\
& \left.\left.+\frac{m a}{(m+\theta)^{3}}\left(e^{-(m+\theta)\left(t_{1}-T\right)}-1\right)-\frac{m a\left(T^{2}-t_{1}^{2}\right)}{2(m+\theta)}\right\}\right] \\
\phi_{20}= & \frac{d \phi_{19}}{d T}=\frac{\phi_{7}}{\left[1+(k-1) e^{-(m+\theta) T}\right]}+t_{1} \frac{d \phi_{7}}{d T}+\phi_{8}+T \frac{d \phi_{8}}{d T}-\frac{d \phi_{9}}{d T} \\
\phi_{21}= & \frac{a}{(m+\theta)}\left[(R-T)-\frac{\left(1-e^{-(m+\theta)(R-T)}\right)}{(m+\theta)}\right] \quad \phi_{22}=\frac{a\left[e^{-(m+\theta)(R-T)}-1\right]}{(m+\theta)} \\
\phi_{23}= & \frac{d \phi_{20}}{d T}=\frac{(k-1) e^{-(m+\theta) T} \phi_{7}}{\left[1+(k-1) e^{-(m+\theta) T}\right]^{2}}+\frac{d \phi_{7}}{\left[1+(k-1) e^{-(m+\theta) T}\right.} \frac{d^{2} \phi_{7}}{d T}+2 \frac{d \phi_{8}}{d T}+T \frac{d^{2} \phi_{8}}{d T^{2}}-\frac{d^{2} \phi_{9}}{d T^{2}}
\end{aligned}
$$


166

$$
\begin{aligned}
& \phi_{24}=\frac{d \phi_{22}}{d T}=a e^{-(m+\theta)(R-T)} \\
& \phi_{25}=\left[\phi_{12}\left(C_{h}+\theta C_{d}\right)+\phi_{13}\left(C_{p}+C\right)+\phi_{15} C_{r} k a+\phi_{24} C_{p} I_{c 1}-\phi_{23} p I_{e}\right] \\
& \phi_{26}=2\left[\phi_{20} p I_{e}-\phi_{5}\left(C_{h}+\theta C_{d}\right)-\phi_{11}\left(C_{p}+C\right)-\phi_{10} C_{r} k a-\phi_{22} C_{p} I_{c 1}\right] \\
& \phi_{27}=2\left[C_{A}+\phi_{1}\left(C_{h}+\theta C_{d}\right)+\phi_{2}\left(C_{p}+C\right)+\phi_{4} C_{r} k a+\phi_{21} C_{p} I_{c 1}-\phi_{19} p I_{e}\right]
\end{aligned}
$$

Appendix 3.

The values are given as follows:

$$
\begin{aligned}
& \phi_{28}=\left[t_{1}\left\{a t_{1}+\frac{m(k-1) a t_{1}}{(m+\theta)}+\frac{m(k-1) a}{(m+\theta)^{2}}\left(e^{-(m+\theta) t_{1}}-1\right)\right\}+T\left\{a\left(S-t_{1}\right)+\frac{m a}{(m+\theta)^{2}}\left(e^{-(m+\theta)\left(t_{1}-T\right)}-e^{-(m+\theta)(S-T)}\right)-\frac{m a\left(S-t_{1}\right)}{(m+\theta)}\right\}\right. \\
& -\left\{\frac{a S^{2}}{2}+\frac{m(k-1) a t_{1}^{2}}{2(m+\theta)}+\frac{m(k-1) a t_{1} e^{-(m+\theta) t_{1}}}{(m+\theta)^{2}}+\frac{m(k-1) a}{(m+\theta)^{3}}\left(e^{-(m+\theta) t_{1}}-1\right)+\frac{m a}{(m+\theta)^{2}}\left(t_{1} e^{-(m+\theta)\left(t_{1}-T\right)}-S e^{-(m+\theta)(S-T)}\right)\right. \\
& \left.\left.+\frac{m a}{(m+\theta)^{3}}\left(e^{-(m+\theta)\left(t_{1}-T\right)}-e^{-(m+\theta)(S-T)}\right)-\frac{m a\left(S^{2}-t_{1}^{2}\right)}{2(m+\theta)}\right\}\right] \\
& \phi_{29}=\frac{d \phi_{28}}{d T}=\frac{\phi_{7}}{\left[1+(k-1) e^{-(m+\theta) T}\right]}+t_{1} \frac{d \phi_{7}}{d T}+\phi_{32}+T \frac{d \phi_{32}}{d T}-\frac{d \phi_{33}}{d T} \\
& \phi_{30}=\frac{a}{(m+\theta)}\left[(S-T)-\frac{\left(1-e^{-(m+\theta)(S-T)}\right)}{(m+\theta)}\right] \quad \phi_{31}=\frac{d \phi_{30}}{d T}=\frac{a\left[e^{-(m+\theta)(S-T)}-1\right]}{(m+\theta)} \\
& \phi_{32}=\left\{a\left(S-t_{1}\right)+\frac{m a}{(m+\theta)^{2}}\left(e^{-(m+\theta)\left(t_{1}-T\right)}-e^{-(m+\theta)(S-T)}\right)-\frac{m a\left(S-t_{1}\right)}{(m+\theta)}\right\} \\
& \phi_{33}=\left\{\frac{a S^{2}}{2}+\frac{m(k-1) a t_{1}^{2}}{2(m+\theta)}+\frac{m(k-1) a t_{1} e^{-(m+\theta) t_{1}}}{(m+\theta)^{2}}+\frac{m(k-1) a}{(m+\theta)^{3}}\left(e^{-(m+\theta) t_{1}}-1\right)+\frac{m a}{(m+\theta)^{2}}\left(t_{1} e^{-(m+\theta)\left(t_{1}-T\right)}-S e^{-(m+\theta)(S-T)}\right)\right. \\
& \left.+\frac{m a}{(m+\theta)^{3}}\left(e^{-(m+\theta)\left(t_{1}-T\right)}-e^{-(m+\theta)(S-T)}\right)-\frac{m a\left(S^{2}-t_{1}^{2}\right)}{2(m+\theta)}\right\} \\
& \frac{d \phi_{32}}{d T}=\left[\frac{a\left\{m(k-1) e^{-(m+\theta) t_{1}}-\theta\right\}}{(m+\theta)\left[1+(k-1) e^{-(m+\theta) T}\right]}-\frac{m a e^{-(m+\theta)(S-T)}}{(m+\theta)}\right] \\
& \frac{d \phi_{33}}{d T}=\frac{m a}{(m+\theta)}\left[\frac{1}{\left[1+(k-1) e^{-(m+\theta) T}\right.}\left\{t_{1}+\frac{e^{-(m+\theta)\left(t_{1}-T\right)}}{(m+\theta)}+\frac{(k-1) e^{-(m+\theta) t_{1}}}{(m+\theta)}\right\}-S e^{-(m+\theta)(S-T)}-\frac{e^{-(m+\theta)(S-T)}}{(m+\theta)}\right] \\
& \frac{d^{2} \phi_{32}}{d T^{2}}=\frac{a(k-1)\left[m(k-1) e^{-(m+\theta)\left(t_{1}+T\right)}-\theta e^{-(m+\theta) T}-m e^{-(m+\theta) t_{1}}\right]}{\left[1+(k-1) e^{-(m+\theta) T}\right]^{2}}-m a e^{-(m+\theta)(S-T)} \\
& \frac{d^{2} \phi_{33}}{d T^{2}}=\frac{m a}{(m+\theta)}\left[\frac{(k-1) e^{-(m+\theta) T}}{\left[1+(k-1) e^{-(m+\theta) T}\right]^{2}}\left\{t_{1}(m+\theta)+e^{-(m+\theta)\left(t_{1}-T\right)}+(k-1) e^{-(m+\theta) t_{1}}+\frac{e^{(m+\theta) T}}{(k-1)}\right\}-(S(m+\theta)+1) e^{-(m+\theta)(S-T)}\right]
\end{aligned}
$$




$$
\begin{aligned}
& \phi_{34}=\frac{d \phi_{29}}{d T}=\frac{(k-1) e^{-(m+\theta) T} \phi_{7}}{\left[1+(k-1) e^{-(m+\theta) T}\right]^{2}}+\frac{2}{\left[1+(k-1) e^{-(m+\theta) T}\right]} \frac{d \phi_{7}}{d T}+t_{1} \frac{d^{2} \phi_{7}}{d T^{2}}+2 \frac{d \phi_{32}}{d T}+T \frac{d^{2} \phi_{32}}{d T^{2}}-\frac{d^{2} \phi_{33}}{d T^{2}} \\
& \phi_{35}=\frac{d \phi_{31}}{d T}=a e^{-(m+\theta)(S-T)} \\
& \phi_{36}=\left[\phi_{12}\left(C_{h}+\theta C_{d}\right)+\phi_{13}\left(C_{p}+C\right)+\phi_{15} C_{r} k a+\phi_{35} C_{p} I_{c 2}-\phi_{34} p I_{e}\right] \\
& \phi_{37}=2\left[\phi_{29} p I_{e}-\phi_{5}\left(C_{h}+\theta C_{d}\right)-\phi_{11}\left(C_{p}+C\right)-\phi_{10} C_{r} k a-\phi_{31} C_{p} I_{c 2}\right] \\
& \phi_{38}=\left[C_{A}+\phi_{1}\left(C_{h}+\theta C_{d}\right)+\phi_{2}\left(C_{p}+C\right)+\phi_{4} C_{r} k a+\phi_{30} C_{p} I_{c 2}-\phi_{28} p I_{e}\right]
\end{aligned}
$$

\section{Acknowledgement}

The second author wish to thank to Council of Scientific and Industrial Research (New Delhi) for providing financial help in the form of JRF vide letter no. 08/017(0017)/2011-EMR-I.

\section{References}

Aggarwal, S. P., \& Jaggi, C. K. (1995). Ordering policies of deteriorating items under permissible delay in payments. Journal of the Operational Research Society, 46(5), 658-662.

Baker, R. C., \& Urban, T. L. (1988). Single-period inventory dependent demand models. Omega, 16(6), 605-615.

Chang, C. T., Chen, Y. J., Tsai, T. R., \& Wu, S. J. (2010). Inventory models with stock and price dependent demand for deteriorating items based on limited shelf space. Yugoslav Journal of Operations Research, 20(1), 55-69.

Chung, K. J., \& Hou, K. L. (2003). An optimal production run time with imperfect production processes and allowable shortages. Computers and Operations Research, 30(4), 483-490.

Covert, R. P., \& Philip, G. C. (1973). An EOQ model for items with Weibull distribution deteriorating. AIIE Transaction, 5(4), 323-326.

Ghare, P. M., \& Schrader, G. F. (1963). A model for exponential decaying inventory. Journal of Industrial Engineering, 14(3), 238-243.

Goyal, S. K. (1985). Economic order quantity under conditions of permissible delay in payments. Journal of the Operational Research Society, 36(4), 335-338.

Goyal, S. K., \& Giri, B. C. (2001). Recent trends in modeling of deteriorating inventory. European Journal of Operational Research, 134(1), 1-16.

Goyal, S. K., \& Barron, L. E. C. (2002). Note on: Economic production quantity model for items with imperfect quality - a practical approach. International Journal of Production Economics, 77(1), 85-87.

Gupta, R., \& Vrat, P. (1986). Inventory model with multi-items under constraint systems for stock dependent consumption rate. Operations Research, 24(1), 41-42.

Hariga, M. A., \& Benkherouf, L. (1994). Optimal and heuristic inventory replenishment models for deteriorating items with exponential time-varying demand. European Journal of Operational Research, 79(1), 123-137.

Kang, F. S., \& Chen, L. H. (2010). Coordination between vendor and buyer considering trade credit and items of imperfect quality. International Journal of Production Economics, 123(1), 52-61.

Khanra, S., Ghosh, S. K., \& Chaudhuri, K. S. (2011). An EOQ model for a deteriorating item with time dependent quadratic demand under permissible delay in payment. Applied Mathematics and Computation, 218(1), 1-9.

Kim, C. H., \& Hong, Y. (1999). An optimal production run length in deteriorating production processes. International Journal of Production Economics, 58(2), 183-189.

Kumar, N., Singh, S. R., \& Kumari, R. (2012). Three echelon supply chain inventory model for deteriorating items with limited storage facility and lead-time under inflation. International Journal of Services and Operations Management, 13(1), 98-118. 
Lee, Y. P., \& Dye, C. Y. (2012). An inventory model for deteriorating items under stock-dependent demand and controllable deterioration rate. Computers \& Industrial Engineering, 63(2), 474-482.

Levin, R. I., McLaughlin, C. P., Lamone, R. P., \& Kottas, J. F. (1972). Production/ Operations Management: Contemporary Policy for Managing Operating Systems. McGraw-Hill, New York.

Mandal, B. N., \& Phaujdar, S. (1989). An inventory model for deteriorating items and stock-dependent consumption rate. Journal of the Operational Research Society, 40(5), 483-488.

Ouyang, L.Y., Wu, K. S., \& Yang, C. T., (2006). A study on an inventory model for non-instantaneous deteriorating items with permissible delay in payments. Computers \& Industrial Engineering, 51(4), $637-651$.

Raafat, F. (1991). Survey of literature on continuously deteriorating inventory models. Journal of the Operational Research Society, 42(1), 27-37.

Ray, J., Goswami, A., \& Chaudhuri, K. S. (1998). On an inventory model with two levels of storage and stock-dependent demand rate. International Journal of Systems Science, 29(3), 249-254.

Rosenblatt, M. J., \& Lee, H. L. (1986). Economic production cycles with imperfect production processes. IIE Transactions, 18(1), 48-55.

Salameh, M. K., \& Jaber, M. Y. (2000). Economic production quantity model for items with imperfect quality. International Journal of Production Economics, 64(3), 59-64.

Sarkar, B. (2012). An EOQ model with delay in payments and stock dependent demand in the presence of imperfect production. Applied Mathematics and Computation, 218, 8295-8308.

Sarkar, B., \& Moon, I. (2011). An EPQ model with inflation in an imperfect production system. Applied Mathematics and Computation, 217(13), 6159-6167.

Shah, V. R., Patel, N. C., \& Shah, D. K. (1988). Economic ordering quantity when delay in payments of order and shortages are permitted. Gujrat Statistical Review, 15(2), 51-56.

Singh, C., \& Singh, S. R. (2011). Imperfect production process with exponential demand rate, Weibull deterioration under inflation. International Journal of Operational Research, 12(4), 430-445.

Singh, S. R., Jain, S., \& Pareek, S., (2012). A warehouse imperfect fuzzified production model with shortages under inflationary conditions. Advances in Decision Sciences, vol. 2012, Article ID 638060, 16 pages.

Singh, S. R., Kumari, R., \& Kumar, N. (2010). Replenishment Policy for non-instantaneous deteriorating items with stock-dependent demand and partial backlogging with two-storage facilities under Inflation. International Journal of Operations Research and Optimization, 1(1), 173-191.

Singh, S. R., Kumari, R., \& Kumar, N. (2011). A deterministic two warehouse inventory model for deteriorating items with stock-dependent demand and shortages under the conditions of permissible delay. International Journal of Mathematical Modelling and Numerical Optimisation, 2(4), 357-375.

Singh, S. R., \& Sharma, S. (2013). A global optimizing policy for decaying items with ramp-type demand rate under two-level trade credit financing taking account of preservation technology. Advances in Decision Sciences, vol. 2013, Article ID 126385, 12 pages.

Singhal, S., \& Singh, S.R. (2013). Volume flexible multi items inventory system with imprecise environment. International Journal of Industrial Engineering Computations, 4(4), 457-468.

Teng, J. T. (2002). On the economic order quantity under conditions of permissible delay in payments. Journal of the Operational Research Society, 53(8), 915-918.

Teng, J. T., Min, J., \& Pan, Q. (2012). Economic order quantity model with trade credit financing for non-decreasing demand. Omega, 40(3), 328-335.

Yang, P. C., \& Wee, H. M., (2006). A Collaborative Inventory System with Permissible Delay in Payment for Deteriorating Items. Mathematical and Computer Modelling, 43(3-4), 209-221.

Yu, J. C. P., Wee, H. M., \& Chen, J. M. (2005). Optimal ordering policy for a deteriorating item with imperfect quality and partial backordering. Journal of the Chinese Institute of Industrial Engineers, 22(6), 509-520.

Zhou, Y. W., \& Yang, S. L. (2005). A two-warehouse inventory model for items with stock-leveldependent demand rate. International Journal of Production Economics, 95(2), 215-228. 